\title{
Osteocyte necrosis triggers osteoclast-mediated bone loss through macrophage-inducible C-type lectin
}

\author{
Darja Andreev, ${ }^{1,2}$ Mengdan Liu, ${ }^{1,2}$ Daniela Weidner, ${ }^{1,2}$ Katerina Kachler, ${ }^{1,2}$ Maria Faas, ${ }^{1,2}$ Anika Grüneboom, ${ }^{1,2}$ \\ Ursula Schlötzer-Schrehardt, ${ }^{3}$ Luis E. Muñoz, ${ }^{1,2}$ Ulrike Steffen, ${ }^{1,2}$ Bettina Grötsch, ${ }^{1,2}$ Barbara Killy, ${ }^{4}$ Gerhard Krönke, ${ }^{1,2}$ \\ Andreas M. Luebke, ${ }^{5}$ Andreas Niemeier, ${ }^{6}$ Falk Wehrhan, ${ }^{7}$ Roland Lang, ${ }^{4}$ Georg Schett, ${ }^{1,2}$ and Aline Bozec ${ }^{1,2}$ \\ 'Department of Internal Medicine 3 - Rheumatology and Immunology, ${ }^{2}$ Deutsches Zentrum für Immuntherapie (DZI), and ${ }^{3}$ Department of Ophthalmology, ${ }^{4}$ Institute of Clinical Microbiology, Friedrich- \\ Alexander-University Erlangen-Nürnberg (FAU) and Universitätsklinikum Erlangen, Erlangen, Germany. ${ }^{5}$ nstitute of Pathology and ${ }^{6}$ Department of Orthopaedics, University Medical Center Hamburg- \\ Eppendorf, Hamburg, Germany. 'Department of Oral and Maxillofacial Surgery, FAU and Universitätsklinikum Erlangen, Erlangen, Germany.
}

\begin{abstract}
Although the control of bone-resorbing osteoclasts through osteocyte-derived RANKL is well defined, little is known about the regulation of osteoclasts by osteocyte death. Indeed, several skeletal diseases, such as bone fracture, osteonecrosis, and inflammation are characterized by excessive osteocyte death. Herein we show that osteoclasts sense damageassociated molecular patterns (DAMPs) released by necrotic osteocytes via macrophage-inducible C-type lectin (Mincle), which induced their differentiation and triggered bone loss. Osteoclasts showed robust Mincle expression upon exposure to necrotic osteocytes in vitro and in vivo. RNA sequencing and metabolic analyses demonstrated that Mincle activation triggers osteoclastogenesis via ITAM-based calcium signaling pathways, skewing osteoclast metabolism toward oxidative phosphorylation. Deletion of Mincle in vivo effectively blocked the activation of osteoclasts after induction of osteocyte death, improved fracture repair, and attenuated inflammation-mediated bone loss. Furthermore, in patients with osteonecrosis, Mincle was highly expressed at skeletal sites of osteocyte death and correlated with strong osteoclastic activity. Taken together, these data point to what we believe is a novel DAMP-mediated process that allows osteoclast activation and bone loss in the context of osteocyte death.
\end{abstract}

\section{Introduction}

The skeleton is a dynamic tissue that undergoes continuous remodeling throughout life, with annual replacement of $10 \%$ of the adult bone. This rather well-understood process is orchestrated by blood-derived monocytes that differentiate into bone-resorbing osteoclasts (1). Bone-resident cells such as osteoblasts and osteocytes synthesize essential osteoclast growth and differentiation factors, including macrophage colony-stimulating factor (M-CSF) and receptor activator of nuclear factor $\kappa-B$ ligand (RANKL) (2-4). An excessive production of pro-osteoclastogenic factors can lead to exaggerated osteoclast activation and pathological bone loss.

Osteocytes are by far the most abundant cells in the bone. During their differentiation from osteoblasts, osteocytes become embedded in the mineralized bone matrix. Osteocytes are located within bony lacunae and are connected to microvessels and to the bone surface via the canalicular network (5). They are well described as RANKL-secreting cells. Conditional deletion of RANKL in osteocytes has demonstrated their importance for the differentiation of osteoclasts (6). As long-lived cells, osteocytes have the ability to regulate organelle damage through the control of autophagy pathways, enabling them to maintain their function (7). However, if the

Conflict of interest: The authors have declared that no conflict of interest exists. Copyright: () 2020, American Society for Clinical Investigation.

Submitted: October 11, 2019; Accepted: June 3, 2020; Published: August 10, 2020

Reference information: / Clin Invest. 2020;130(9):4811-4830.

https://doi.org/10.1172/JCl134214. damage is excessive, osteocytes undergo cell death. It is considered that the disruption of canalicular flow, in the context of bone damage, causes an undersupply of osteocytes with essential nutrients and oxygen (8). Osteocyte death was initially considered programmed cell death by apoptosis (9). However, because phagocytes cannot reach and engulf apoptotic osteocytes in their isolated lacunae, these cells automatically undergo secondary necrosis, losing their membrane integrity and releasing damage-associated molecular patterns (DAMPs) into their environment (10).

Several bone diseases are associated with enhanced osteocyte death. For instance, it has been demonstrated that TNFmediated arthritis is accompanied by a high number of empty osteocyte lacunae (11). In addition, arthritic synovium is characterized by the presence of various DAMPs (12). Massive bone damage, caused by fracture, also induces osteocyte death (13) and substantial accumulation of mitochondrial DAMPs (14). In addition, osteocyte death is most strongly linked to the pathogenesis of osteonecrosis, a disease that is characterized by the death of bone tissue in the absence of regeneration, resulting in a collapse of necrotic bone (15). Although it is known that osteocyte death and bone damage trigger osteoclast formation and bone loss, the underlying mechanism of this process is still unclear. This process appears to be different from cytokine-induced osteoclast differentiation, because dead osteocytes are no longer capable of cytokine synthesis. However, necrotic osteocytes release large amounts of DAMPs, which might present a hitherto undiscovered process of osteoclast activation. 
A

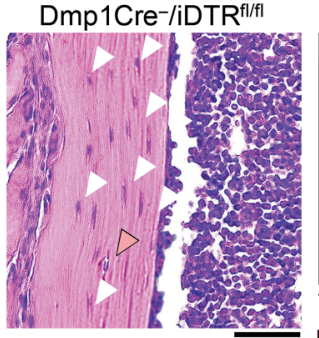

2.04\% Empty lacunae

$\square 8.65 \%$ Dying osteocytes

$\square 89.31 \%$ Filled lacunae

B

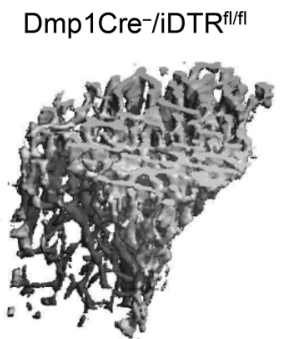

Dmp1Cre+/iDTR ${ }^{\text {fl/f }}$

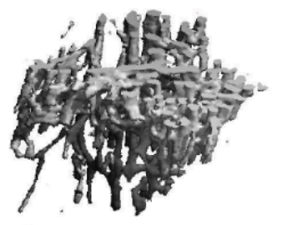

D

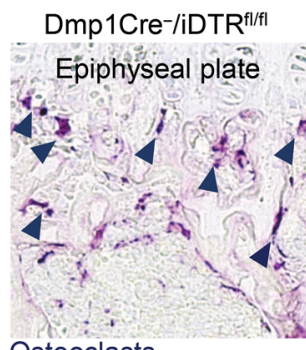

Osteoclasts

$\mathbf{F}$
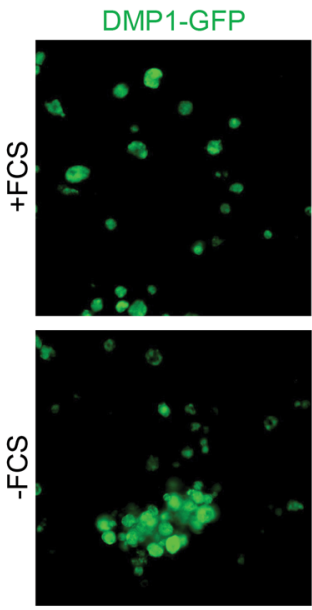

Dmp1Cre+/iDTR $\mathrm{fl/f}$

Epiphyseal plate
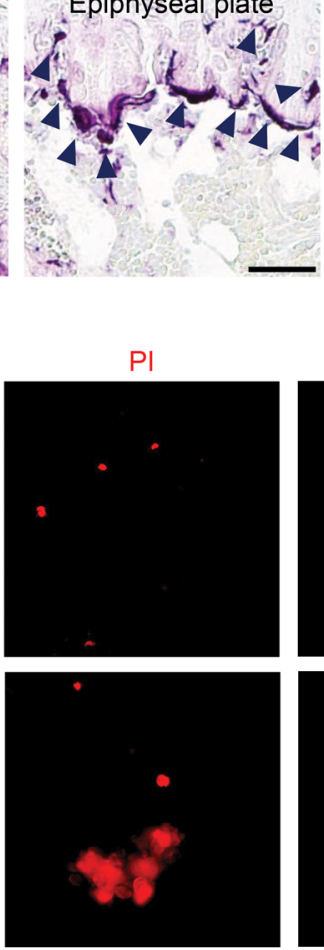

Total $=100(n=10)$

Dmp1Cre $/ / \mathrm{DTR}^{\mathrm{ff} / \mathrm{fl}}$

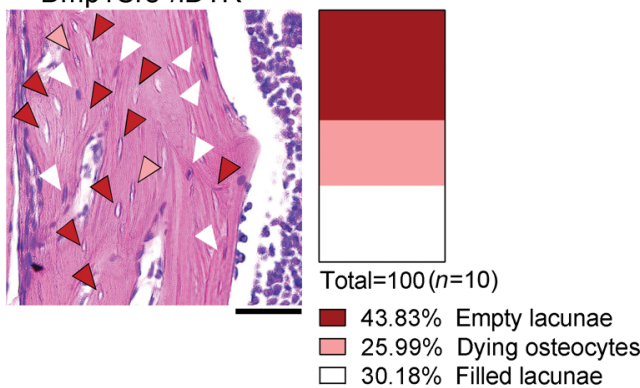

C

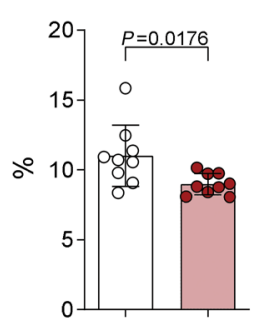

Tb.N

Tb.Sp

Tb.Th

- Dmp1Cre-/iDTR ${ }^{f l / f i}(n=9)$
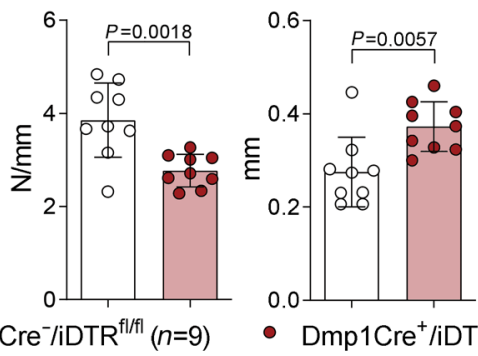

- Dmp1Cre ${ }^{+} /$iDTR $^{\mathrm{fl} / \mathrm{lif}}(n=9)$
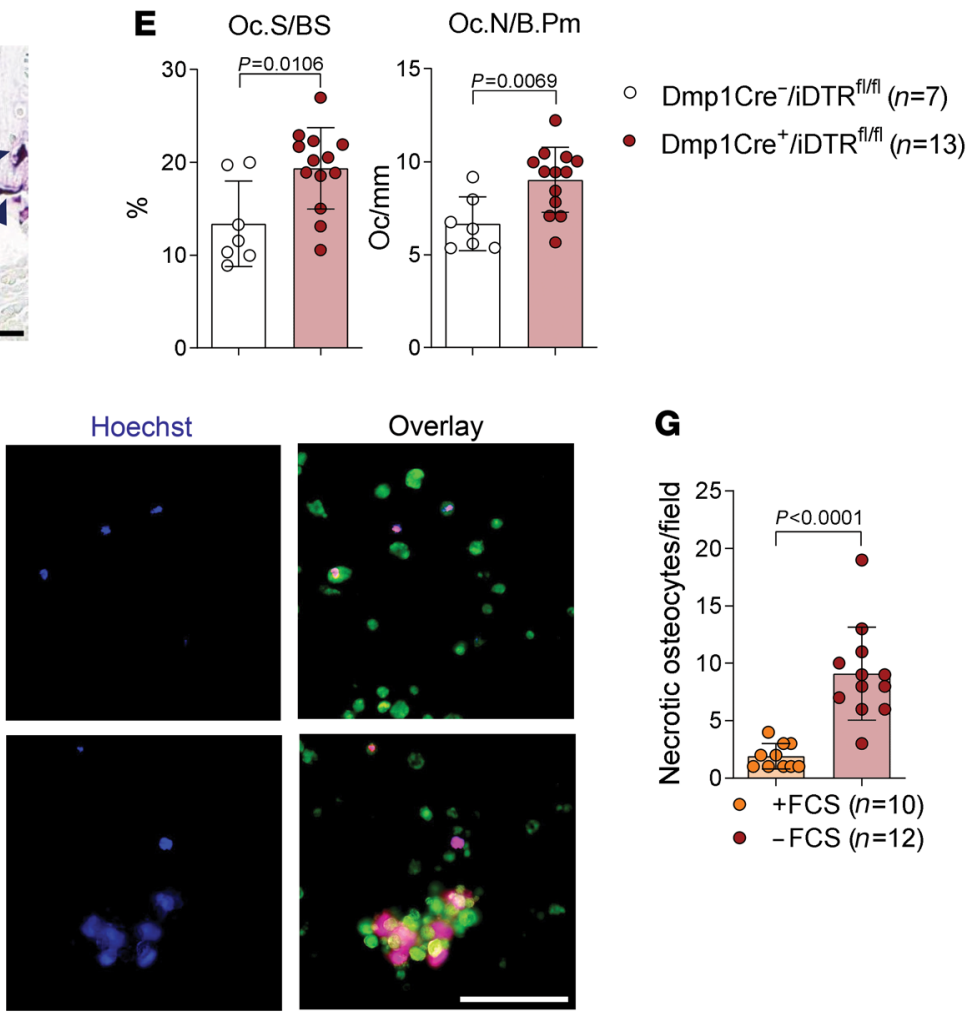

G

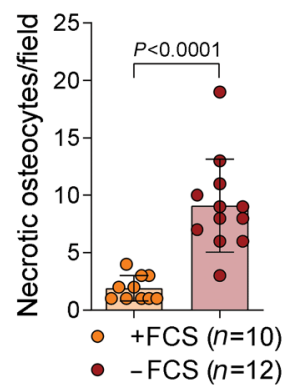

H

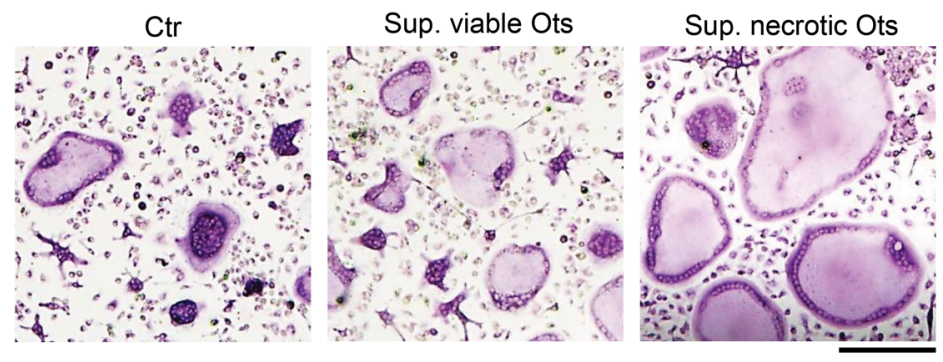

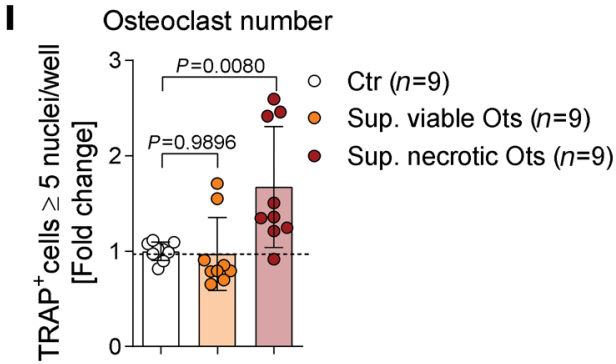


Figure 1. Osteocyte necrosis triggers osteoclastogenesis. (A) Representative pictures of hematoxylin and eosin (H\&E) staining of tibial bones showing induced ablation of osteocytes in 9-week-old Dmp1-Cre ${ }^{+} / \mathrm{iDTR}^{\mathrm{fl} /}$ fl mice compared with Dmp1-Cre-/iDTR ${ }^{\mathrm{fl} / \mathrm{fl}}$ littermate controls, 4 days after i.p. injection with $100 \mathrm{ng}$ diphtheria toxin (DT) ( $n=10$ /group). White arrows indicate filled lacunae, orange arrows dying osteocytes, and red arrows empty lacunae. Scale bars: $50 \mu \mathrm{m}$. (B) Representative micro-computed tomography $(\mu \mathrm{CT})$ images of $\mathrm{Dmp1}-\mathrm{Cre}^{+} / \mathrm{iDTR}^{\mathrm{f} / / \mathrm{fl}}$ tibias compared with Dmp1-Cre $/$ iDTR $^{\mathrm{fl} / \mathrm{fl}}$ littermate controls and (C) quantification of bone volume per total volume (BV/TV), trabecular number (Tb.N), trabecular separation (Tb.Sp), and trabecular thickness (Tb.Th) ( $n=9 /$ group). Scale bar: $1 \mathrm{~mm}$. (D) Representative TRAP staining in tibial sections of the aforementioned 2 groups. Dark blue arrows indicate the purple-stained osteoclasts. Scale bar: $50 \mu \mathrm{m}$. (E) Histomorphometric quantification of osteoclast surface per bone surface (Oc.S/BS) and osteoclast number per bone perimeter (Oc.N/B.Pm) in the tibia of the aforementioned 2 groups ( $n=7-13 /$ group). (F) Representative images of DMP1-GFP-positive (green) starved osteocytes (24 hours without fetal calf serum, FCS) compared with viable osteocytes (24 hours with FCS), stained for necrosis with PI (red) and Hoechst (blue). Scale bar: $100 \mu \mathrm{m}$. (C) Quantification of necrotic osteocytes after serum starvation (24 hours) compared with serum-supplemented controls ( $n=10-12$ /group). (H) Representative pictures and (I) quantification of TRAP-positive polynucleated ( $\geq 5$ nuclei) WT osteoclasts, supplemented with supernatant from viable (+FCS) or necrotic (-FCS) osteocytes (Ots) $(1: 2)$ on day 1 of culture for 24 hours, compared with a nonsupplemented control ( $n=9$ /group). Scale bar: $200 \mu \mathrm{m}$. Data are shown as mean \pm SD. $P$ values were determined by 2-tailed Student's $t$ test for single comparisons (C, E, and $\mathbf{G}$ ) or 1-way ANOVA for multiple comparisons (I).

DAMPs are recognized by pattern recognition receptors (PRRs). These receptors are mainly expressed on macrophages, monocytes, dendritic cells, and neutrophils (16). Key DAMP-binding PRRs include advanced glycosylation end-product-specific receptor (AGER), Toll-like receptors (TLRs), and C-type lectin receptors (17). The PRR macrophage-inducible C-type lectin (Mincle) is unique, since it is not only able to sense pathogen-associated molecular patterns (PAMPs) but also several DAMPs (18). Mincle is traditionally associated with the defense against fungal and bacterial infections (19). Its most prominent ligand is the mycobacterial cord factor trehalose-6,6'-dimycolate (TDM) $(19,20)$. Recent findings, however, suggest that Mincle is also involved in tissue homeostasis (21). Mincle senses the endogenous molecules Sin3A-associated protein 130 (SAP-130) and $\beta$-glucosylceramide ( $\beta$-GlcCer), which are specifically released upon necrotic cell death $(22,23)$. Interestingly, Mincle possesses a signal transduction pathway similar to the one from the osteoclast costimulators OSCAR (osteoclast associated, immunoglobulin-like receptor) and PIR-A (paired Ig-like receptor-A). It acts via the immunoreceptor tyrosine-based activation motif-harboring (ITAM-harboring) adaptor Fc receptor common $\gamma$ subunit $(\mathrm{FcR} \gamma)$, leading to the activation of the spleen tyrosine kinase (SYK)/calcium axis $(22,24)$. We therefore hypothesized that Mincle may represent the missing link between osteocyte death and osteoclast activation.

\section{Results}

Necrotic osteocytes induce osteoclastogenesis. To delineate whether osteocyte death could trigger an in vivo bone phenotype, a genetic diphtheria toxin-based (DT-based) inducible osteocyte ablation was established (25). To do so, Dmp1-Cre mice carrying the gene for the DT receptor (DTR) subsequent to a loxP-flanked stop codon were generated. Dentin matrix protein 1 (DMP1) is almost exclusively expressed in mature osteocytes $(26,27)$. Thus, in Dmp1-Cre $/ \mathrm{iDTR}^{\mathrm{A} / \mathrm{fl}}$ mice all osteocytes express the DTR on their surface and the administration of DT leads to osteocyte death, while other cells remain intact. As expected, an increased number of dying osteocytes and empty lacunae were observed in the long bones of Dmp1-Cre ${ }^{+} / \mathrm{iDTR}^{\mathrm{f} / \mathrm{l} / \mathrm{l}}$ mutant mice, as compared with Dmp1-Cre $-/ \mathrm{iDTR}^{\mathrm{A} / \mathrm{fl}}$ negative controls (Figure 1A). Moreover, the DT-induced cell death of osteocytes resulted in an osteoporotic bone phenotype (Figure 1, B and C), as indicated by an increase in the osteoclast-covered surface and osteoclast numbers in the long bones (Figure 1, D and E).

To define, in principle, whether necrotic and apoptotic cells have distinct effects on osteoclast differentiation, WT osteoclasts were stimulated with necrotic versus apoptotic splenocytes. Osteoclasts were differentiated from bone marrow-derived monocytes (BMMs) in the presence of $20 \mathrm{ng} / \mathrm{mL}$ $\mathrm{M}-\mathrm{CSF}$ and $10 \mathrm{ng} / \mathrm{mL}$ RANKL within 4-5 days. On day 1 of osteoclastogenesis, preosteoclasts were stimulated with necrotic versus apoptotic cells for 24 hours. Interestingly, we detected increased mRNA expression of osteoclast-specific markers and an elevated number of tartrate-resistant acid phosphatase-positive (TRAP-positive) osteoclasts only in the context of stimulation with necrotic cells (Supplemental Figure 1, A-C; supplemental material available online with this article; https://doi. org/10.1172/JCI134214DS1). In contrast with necrotic cells, apoptotic cells did not induce osteoclast activation (Supplemental Figure 1, A-C). To translate these findings to osteocyte death, we made use of the osteocytic cell line IDG-SW3 (Supplemental Figure 1D) that undergoes necrosis after serum starvation (Figure 1, F and G, and Supplemental Video 1). Although the supernatant of viable osteocytes had no effect on osteoclast differentiation, the supernatant of necrotic osteocytes strongly enhanced osteoclastogenesis (Figure 1, H and I). These data show that components released by necrotic osteocytes specifically enhance osteoclasts in vivo and in vitro.

Osteoclasts sense necrotic osteocytes via the C-type lectin receptor Mincle. DAMP release is a hallmark of necrotic cell death. The C-type lectin receptor Mincle is described as a sensor for several DAMPs, in particular the small nuclear ribonucleoprotein SAP-130 $(22,28)$. We investigated the level of SAP-130 in the supernatant of viable, apoptotic or necrotic osteocytes compared with necrotic lysates from splenocytes or osteocytes. Indeed, SAP-130 was specifically released by osteocytes undergoing necrosis (Figure 2A). Thereupon, we analyzed the mRNA expression level of several PRRs that are known to recognize DAMPs, including AGER, TLR2, TLR4, and Mincle (encoded by the gene Clec4e) in osteoclast cultures after stimulation with viable or necrotic osteocytes. As shown in Figure 2B, only the expression of Mincle was specifically upregulated in osteoclasts after addition of necrotic osteocytes. These results suggest that DAMPs from necrotic osteocytes boost osteoclastogenesis via a Mincle-dependent mechanism.

We next compared the expression pattern of Mincle in bone marrow-derived (BM-derived), in vitro-differentiated murine osteoclasts with the expression level in total BM cells and osteoblasts, differentiated from calvarial osteoprogenitors. Cellular purity of osteoblasts was verified by the expression of the osteo- 
A

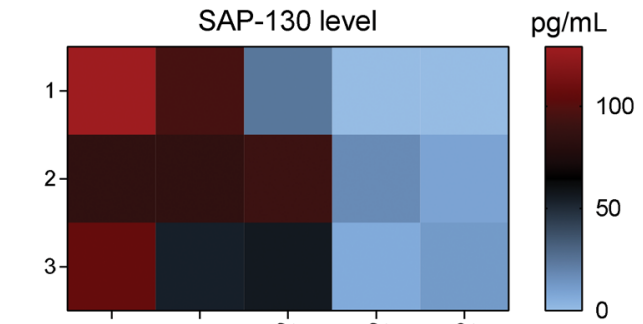

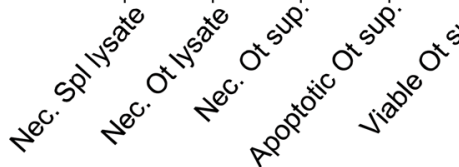

C

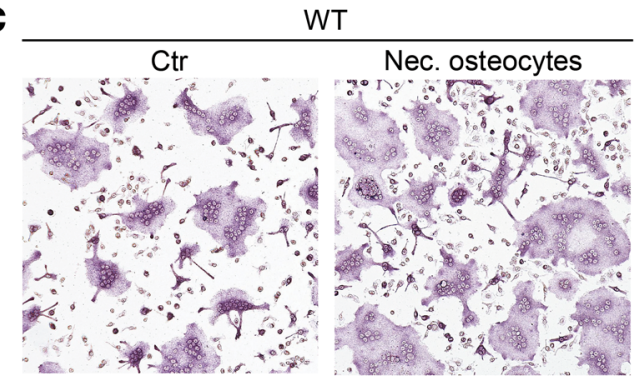

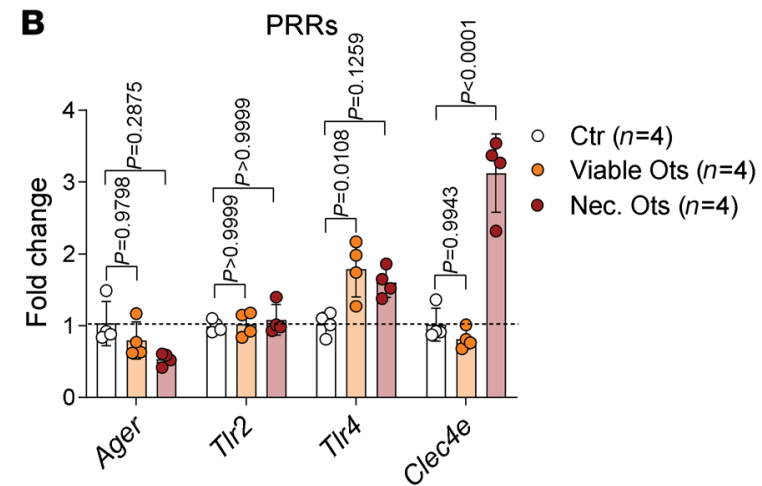

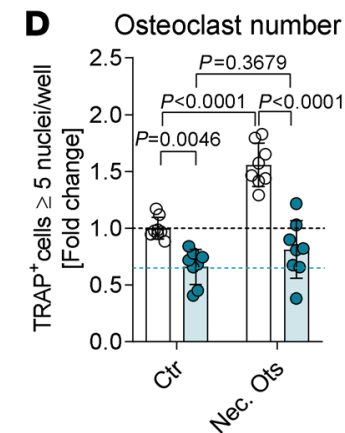

- WT $(n=8) \circ$ Mincle $\mathrm{KO}(n=8)$

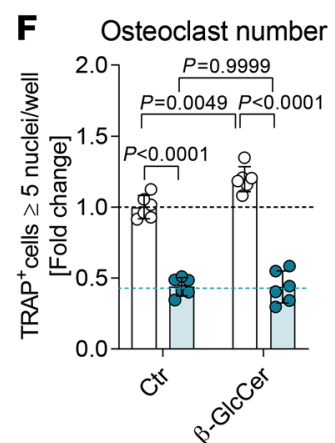

- WT $(n=6) \circ$ Mincle KO $(n=6)$
E

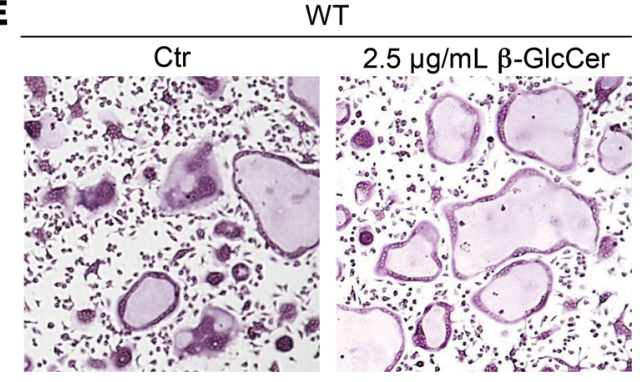

Mincle KO

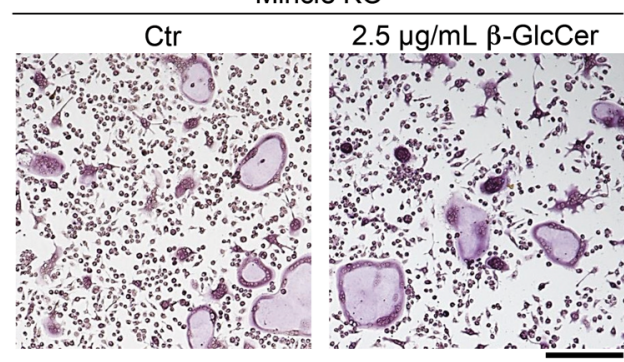

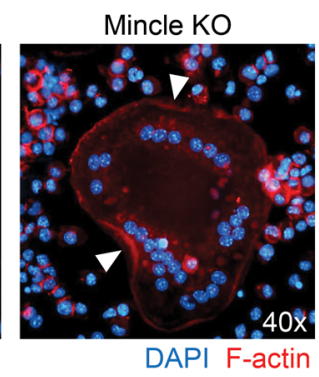

WT $(n=15)$

- Mincle $\mathrm{KO}(n=15)$

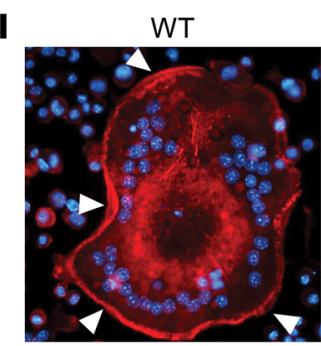

DAPI F-actin
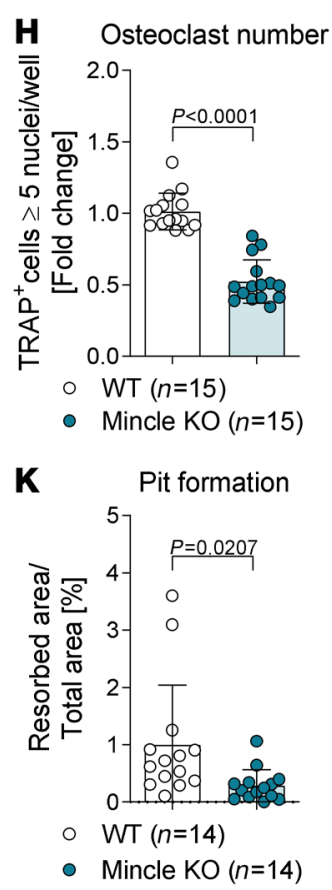

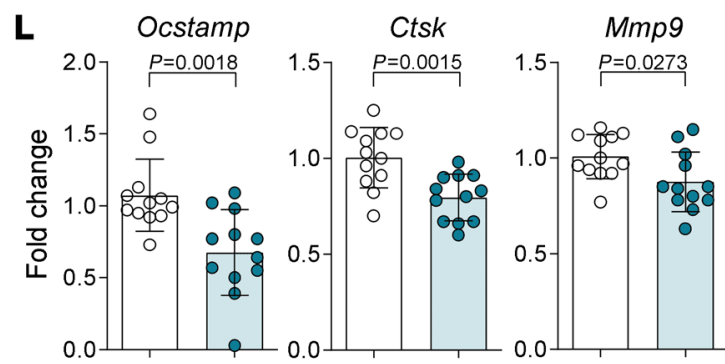

○ WT $(n=12)$ Mincle KO $(n=12)$ 
Figure 2. Necrotic osteocyte-derived DAMPs induce osteoclastogenesis in a Mincle-dependent manner. (A) Heatmap of the SAP-130 levels in lysates of necrotic splenocytes and osteocytes (Ots) and in the supernatants of viable, apoptotic, and starved osteocytes ( $n=3 /$ group). (B) Gene expression of Ager, TIr2, TIr4, and Clec4e in WT osteoclasts, stimulated with viable or necrotic osteocytes (1:2) on day 1 of culture for 24 hours, compared with unstimulated control ( $n=4 /$ group). (C) Representative images and (D) quantification of TRAP-positive polynucleated ( $\geq 5$ nuclei) WT and Mincle-KO osteoclasts, supplemented with necrotic osteocytes (1:2) for 24 hours on day 1 of culture, compared with unstimulated controls ( $n=8 /$ group). Scale bar: $200 \mu \mathrm{m}$. (E) Representative pictures and (F) quantification of TRAP-positive polynucleated ( $\geq 5$ nuclei) WT and Mincle-KO osteoclasts, supplemented with $2.5 \mu \mathrm{g} / \mathrm{mL} \beta$-glycosylceramide ( $\beta$-GlcCer) for 24 hours on day 1 of culture, compared with nonsupplemented controls ( $n=6 /$ group). Scale bar: $200 \mu \mathrm{m}$. (G) Representative pictures and $(\mathbf{H})$ quantification of TRAP-positive polynucleated ( $\geq 5$ nuclei) osteoclasts derived from Mincle-KO compared with WT mice ( $n=15$ / group). Scale bar: $200 \mu \mathrm{m}$. (I) Immunofluorescence microscopy of WT and Mincle-KO osteoclasts, stained with DAPI (blue) and for F-actin (red). White arrows illustrate the F-actin ring. Original magnification, $\times 40$. (J) Representative pictures of resorption assay and $(\mathbf{K})$ quantification of the percentage of the resorbed area by Mincle-KO compared with WT osteoclasts ( $n=14 /$ group). Scale bar: $200 \mu \mathrm{m}$. (L) Gene expression analysis of Ocstamp, Ctsk, and Mmpg in Mincle-KO compared with WT osteoclasts ( $n$ $=12$ (group). Data are shown as mean \pm SD. $P$ values were determined by 2-way ANOVA for multiple comparisons (B, D, and $\mathbf{F}$; interaction $P$ value: $<0.0001$ for B, 0.0038 for $\mathbf{D}$, and 0.0116 for $\mathbf{F}$ ) or 2-tailed Student's $t$ test for single comparisons ( $\mathbf{H}, \mathbf{K}$, and $\mathbf{L}$ ).

calcin gene Bglap (Supplemental Figure 2A). Clec4e expression was 3 times higher in osteoclasts than in BM cells and was undetectable in osteoblasts (Supplemental Figure 2A). Furthermore, osteoclast lineage cells expressed Clec $4 e$ predominantly on day 3 of differentiation, which falls together with the expression peak of early osteoclast markers (Supplemental Figure 2, B and C). Moreover, immunofluorescence costaining of Mincle, osteoclast marker TRAP, and DAPI staining (for nuclei detection) showed Mincle expression only in mono- and binucleated preosteoclasts (Supplemental Figure 2D). These data indicate that Mincle is upregulated during early osteoclast differentiation.

To determine the function of Mincle during osteoclast formation and activity, WT and Mincle-deficient preosteoclasts were stimulated with necrotic osteocytes. In line with our previous findings, necrotic osteocytes strongly induced osteoclastogenesis (Figure 2, C and D). In contrast, however, Mincle-knockout (Mincle-KO) osteoclasts were unable to respond to necrotic osteocytes (Figure 2, C and D). Furthermore, we tested osteoclast stimulation with the intracellular metabolite $\beta$-glucosylceramide ( $\beta$-GlcCer), a DAMP ligand for Mincle, which is located in the endoplasmic reticulum/Golgi apparatus of the cell and is released into the extracellular milieu upon necrotic cell death (23). Stimulation with $\beta$-GlcCer was able to induce osteoclastogenesis in a Mincle-dependent manner (Figure 2, E and F). To a similar extent, another Mincle agonist, trehalose-6,6'-dibehenate (TDB), led to a dose-dependent increase in osteoclast numbers and size (Supplemental Figure 2, E and F). As expected, the addition of a neutralizing anti-Mincle antibody inhibited osteoclastogenesis in a dose-dependent way (Supplemental Figure 2, G and H). Thus, Mincle on preosteoclasts senses dead osteocytes and as a response induces osteoclast activation.
We additionally comparatively characterized the osteoclastogenic potential of Mincle-competent (WT) and -deficient (-KO) osteoclast lineage cells. In line with the above findings, the ability of BM-derived monocytes to differentiate into large polynucleated osteoclasts was diminished in the absence of Mincle (Figure 2, G and $\mathrm{H})$. Mincle operates independently of RANKL signaling, since WT and Mincle-KO cells showed similar upregulation of osteoclastogenesis with increasing RANKL concentration, although the overall number of Mincle-KO osteoclasts was reduced (Supplemental Figure 3, A and B). Moreover, we examined the bone resorbing activity of WT and Mincle-KO osteoclasts. Osteoclast activity depends on the formation of the sealing zone, a dynamic actin-rich ring structure, which attaches osteoclasts to the bone surface and delimits the resorption lacunae, containing all the proteolytic enzymes (1). Fluorescence-based staining of $\mathrm{F}$-actin revealed that Mincle-KO osteoclasts had decreased actin ring formation compared with WT osteoclasts (Figure 2I). Furthermore, Mincle-KO osteoclasts exhibited a strongly reduced capacity to resorb synthetic bone in comparison with WT osteoclasts (Figure 2, J and K). The diminished osteoclast number and activity can be explained by a reduced mRNA expression of the osteoclast fusion regulator, osteoclast-stimulatory transmembrane protein (OC-STAMP), and the matrix enzymes cathepsin $\mathrm{K}$ (CTSK) and matrix metalloproteinase 9 (MMP9), in Mincle-KO osteoclasts as compared with WT osteoclasts (Figure 2L).

DAMP/Mincle axis alters osteoclast gene networks and skews the metabolic activity of osteoclasts into oxidative phosphorylation. To understand the mechanistic role of Mincle in osteoclasts, we performed in-depth analysis of the effect of Mincle deficiency on the genomic transcriptional network of osteoclasts. Whole-transcriptome RNA sequencing (RNA-seq) was performed on osteoclasts stimulated with necrotic osteocyte supernatant compared with control osteoclasts, derived from either WT or Mincle-KO mice. Differential expression analysis revealed that loss of Mincle in osteoclasts significantly upregulated 7 genes, while downregulating 9 (Figure 3A). Interestingly, upon stimulation with necrotic osteocyte supernatant, Mincle deficiency had a more decisive impact on the transcriptional network of osteoclasts, leading to a significant upregulation of 48 genes and downregulation of 18 genes (Figure 3A). Several of the downregulated genes are associated with calcium signaling, i.e., Camk2b and $C n b d 2$, and cell metabolism (Figure 3B). In addition, KEGG pathway enrichment analyses revealed that among the downregulated pathways, oxidative phosphorylation and calcium signaling were strongly affected (Figure 3C). Downregulation of the "tuberculosis" pathways validates our data, since Mycobacterium tuberculosis is recognized by Mincle. The predicted pathway indicates that Mincle acts upstream of the FcR $\gamma /$ SYK axis (Supplemental Figure 4).

To verify the hypothesis that DAMP-mediated Mincle engagement might change the metabolic activity of osteoclasts, we performed an extracellular flux assay comparing the oxidative phosphorylation in WT and Mincle-deficient osteoclasts upon stimulation with necrotic osteocyte supernatant. Indeed, Mincle was necessary to induce mitochondrial respiration in osteoclasts when exposed to osteocytic DAMPs (Figure 4, A and B). This observation was confirmed by the Mincle-dependent upregulation of the oxidative phosphorylation-related genes Sdha (succinate dehydrogenase complex flavoprotein subunit A), $S d h b, S d h c$, 
A

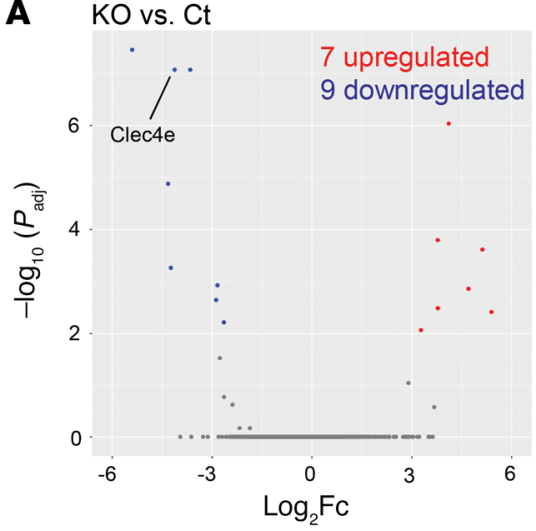

KONecOt vs. CtNecOt

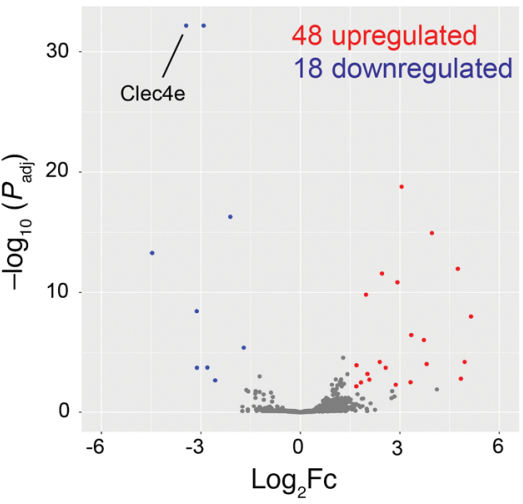

C

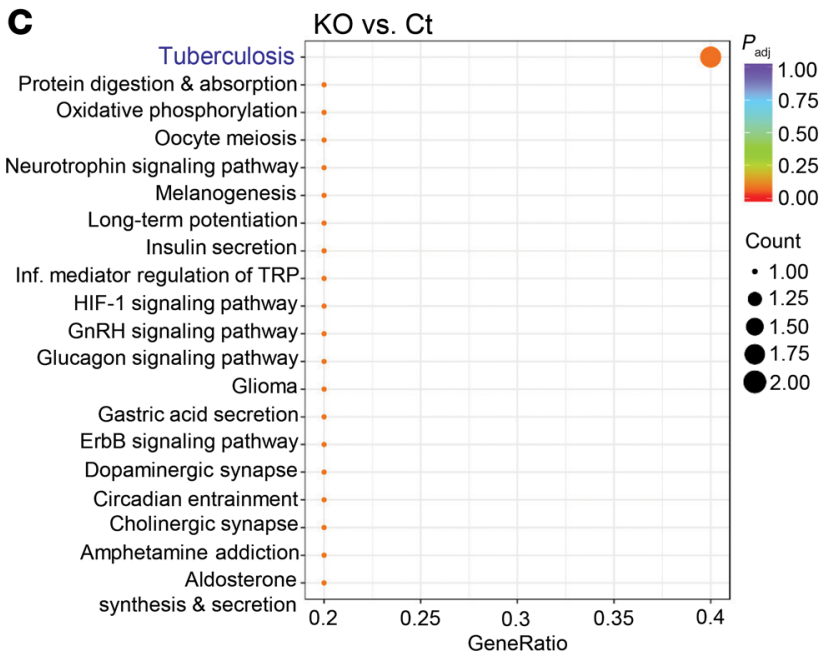

B

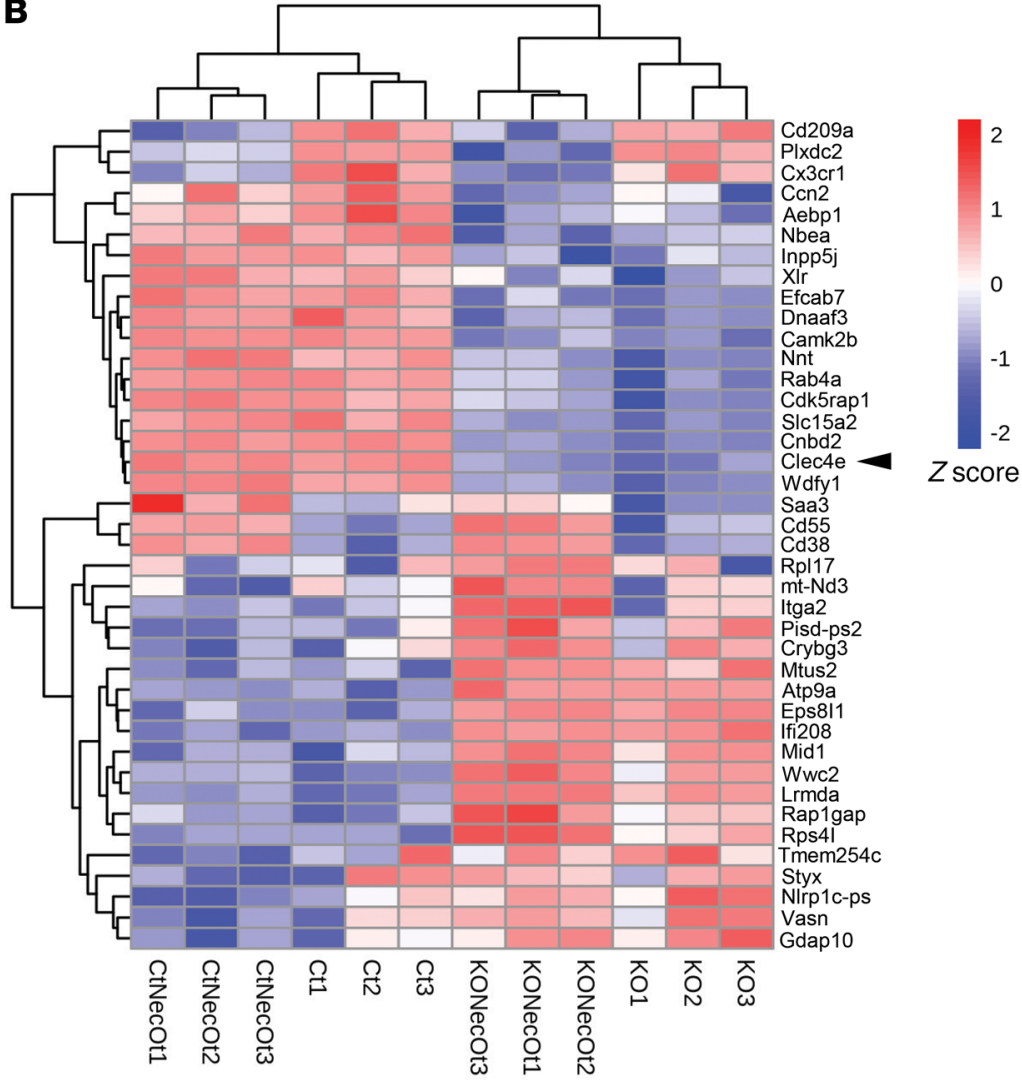

Figure 3. DAMP/Mincle axis alters osteoclast gene networks affecting calcium signaling and cell metabolism. RNA sequencing was performed with the following 4 groups (3 replicates per group): Ct (WT osteoclasts, control condition); KO (Mincle-KO osteoclasts, control condition); CtNecOt (WT osteoclasts stimulated on day 1 for 24 hours with 1:2 necrotic osteocyte supernatant); and KONecOt (Mincle-KO osteoclasts stimulated on day 1 for 24 hours with 1:2 necrotic osteocyte supernatant). (A) Volcano plots showing the altered gene expression between the groups KO vs. Ct and KONecOt vs. CtNecOt. Statistically significantly upregulated genes are red and downregulated genes are blue. (B) Heatmap showing the H-clusters of 40 differentially expressed genes between the aforementioned 4 groups. (C) KEGG enrichment analysis showing significantly affected pathways, comparing the groups KO vs. Ct and KONecOt vs. CtNecOt. Genes with an adjusted $P$ value $\left(P_{\text {adj }}\right)$ less than 0.05 were assigned as differentially expressed. Pathways with $P_{\text {adj }}$ less than 0.05 were considered significantly enriched.

and Sdhd upon exposure to necrotic osteocytes (Figure 4C). In accordance, transmission electron microscopy (TEM) imaging of WT osteoclasts revealed a sharp increase in the number of mitochondria upon stimulation with necrotic osteocytes, which was much less pronounced in Mincle-deficient cells (Figure 4D).
To investigate whether Mincle is essential for the ability of osteoclasts to sense necrotic cell death, we monitored movements of WT and Mincle-deficient osteoclasts upon stimulation with necrotic osteocytes. Although the speed and moving distance were not dependent on Mincle expression, the movement 
direction toward the necrotic cells was impaired in Mincle-deficient osteoclasts (Figure 4, E and F, and Supplemental Figure 5A, and Supplemental Video 2). As monocyte-derived cells, osteoclasts have the potential to engulf dead cells (29). However, Mincle deficiency had no influence on the phagocytic capacity of osteoclasts to take up E. coli BioParticles or necrotic cells (Supplemental Figure 5, B-G). Hence, Mincle activation through necrotic cells skews the metabolic activity of osteoclasts toward higher oxidative phosphorylation, thereby enhancing their differentiation. In addition, Mincle controls the directed migration of osteoclasts toward dead cells.

Mincle-deficient mice exhibit higher bone mass due to reduced osteoclast numbers. Our in vitro studies showed that Mincle influences osteoclast formation and activity. Immunofluorescence staining of Mincle in combination with the osteoclast lineage marker CD68 (30) in long bones of WT mice revealed Mincle-positive osteoclasts attached to the bone surface (Supplemental Figure 6A), suggesting an in vivo role for Mincle in bone homeostasis. Therefore, we performed 3-dimensional bone structure analyses on tibial bones from 9-week-old and 5-month-old Mincle-KO and WT mice by micro-computed tomography $(\mu \mathrm{CT})$. In Mincle-KO mice, bone mass was significantly increased compared with WT mice, as represented by elevated bone volume and trabecular number, while the trabecular separation was accordingly reduced (Figure 5, A and B, and Supplemental Figure 6, B and C). Similar observations were made in 5-month-old mice, showing elevated bone mass in Mincle-deficient animals (Supplemental Figure 6, D and E).

To test whether the high bone mass phenotype of Mincle-KO mice indeed stems from reduced osteoclast activity or may involve changes in the osteoblast compartment, we performed histomorphometric analyses of toluidine blue-stained tibial bone sections. Number and distribution of osteoblasts on the bone surface were similar in Mincle-KO and WT mice (Supplemental Figure 7, A and B). The serum levels of osteoblast-derived cytokines, such as RANKL, osteoprotegerin (OPG), osteopontin (OPN), and osteocalcin were also not altered by Mincle loss (Supplemental Figure 7C). Furthermore, no difference was detected regarding the number and distribution of osteocytes within the bone matrix (Supplemental Figure 7D). The levels of the osteocyte-related cytokines sclerostin and dickkopf 1 (DKK-1) were the same in the serum of Mincle-KO and WT mice (Supplemental Figure 7E). To further analyze the activity of osteoblasts, calcein injections and measurement of bone formation parameters were carried out. Mincle-KO mice showed no difference in osteoblast-dependent bone formation (Supplemental Figure 7, F and G). Taken together, the osteoblast/osteocyte compartment was not affected by the absence of Mincle, suggesting that Mincle controls bone mass through the regulation of in vivo osteoclast function.

The number of macrophages and osteoclast precursors was not altered in the blood of Mincle-KO as compared with WT mice (Supplemental Figure 7, H-K), indicating a normal development of the myeloid lineage and sufficient mobilization of osteoclast precursors in Mincle-KO mice. However, TRAP staining of tibial bone sections showed a strongly decreased osteoclast number and osteoclast-covered bone surface in Mincle-KO as compared with WT controls (Figure 5, C and D). In line with our in vitro data, these results point toward a differentiation defect, affecting the fusion of precursors into polynucleated osteoclasts. Taken together, our results indicate that Mincle-KO mice exhibit increased bone mass due to a reduced number of osteoclasts in the long bones.

Mincle selectively influences bone diseases linked to osteocyte death. Osteoclast activation, but not necessarily osteocyte death, is a hallmark of postmenopausal osteoporosis. Therefore, we examined the influence of Mincle on bone loss following estrogen withdrawal. We performed ovariectomy in female mice (31), which resulted in equal weight gain and uterus shrinkage in WT and Mincle-KO mice (Supplemental Figure 8, A-C). Despite starting from higher bone mass at baseline, Mincle-KO mice showed a similar level of bone loss after ovariectomy to that observed in WT mice (Supplemental Figure 8, D and E). Ovariectomy-induced increases in osteoclast number and osteoclast-covered bone surface were less pronounced in Mincle-KO mice (Supplemental Figure 8, F and G); however, bone loss still occurred in the absence of Mincle, suggesting that Mincle is not a key element in bone loss following estrogen deficiency.

Unlike estrogen deficiency, rheumatoid arthritis (RA), a chronic inflammatory disease of the joints, is characterized by low bone formation, osteocyte death, and osteoclast activation, as shown by in-depth analyses of human bone from RA patients (32). We therefore tested a model of arthritis-induced bone loss by exposing WT and Mincle-KO mice to arthritogenic K/BxN serum (33). Mincle-KO mice developed a stronger local inflammation than WT mice (Supplemental Figure 9A), probably due to an altered inflammatory macrophage response (Supplemental Figure 9, B and C). In sharp contrast, bone parameters in the tibia were better preserved in Mincle-KO mice than in WT controls upon seruminduced arthritis (SIA) (Figure 5, E and F). In accordance with the previous data, higher systemic bone mass of Mincle-KO mice after SIA was associated with reduced number of TRAP-positive osteoclasts, covering a smaller area of bone (Figure 5, G and H).

Another bone disease, characterized by osteocyte death, is fracture $(5,8)$. Thus, we hypothesized that Mincle could play a role in fracture repair. Indeed, high numbers of empty osteocyte lacunae were clearly detectable in the fractured compared with the normal, nonfractured bone compartment, 14 days after fracture (Figure 6A). In addition, a highly increased number of CD68 ${ }^{+}$Min$\mathrm{cle}^{+}$cells was observed in fractured compared with normal bone (Figure 6, B and C). The repair mechanism upon fracture is composed of different phases, starting with a hematoma formation, followed by the formation of a soft callus, which is then replaced by bone (34). The mobility and weight loss upon femur fracture were comparable in WT and Mincle-KO mice (Supplemental Figure 10A). Both animal groups showed unrestricted bone loading a few days after femoral fracture. Interestingly, Mincle-KO mice developed a larger and denser callus compared with WT mice, 14 days after fracture (Figure 6, D-F). In addition, the callus of Mincle-KO mice was characterized by strikingly increased vascularization (Figure 6E and Supplemental Video 3). Furthermore, 21 days after fracture, the fractured bone ends were better connected in Mincle-KO mice than in WT mice (Supplemental Figure 10, B and C). Mincle-KO mice were able to heal the femoral fracture completely after 3 months, which was comparable to WT animals (Supplemental Figure 10, D and E). In line with the previous results, the improved fracture repair was mediated by a reduced number of 
A Oxidative phosphorylation

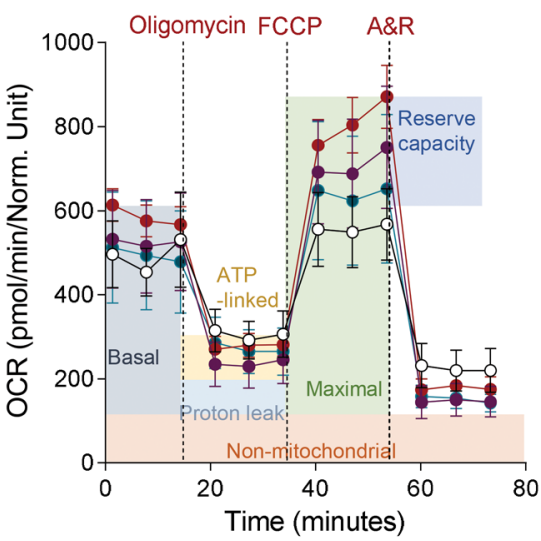

-o- WT $(n=4)$

$\bullet W T+$ Nec. Ot sup. $(n=4)$

- Mincle KO $(n=4)$

- Mincle KO + Nec. Ot sup. $(n=4)$
B

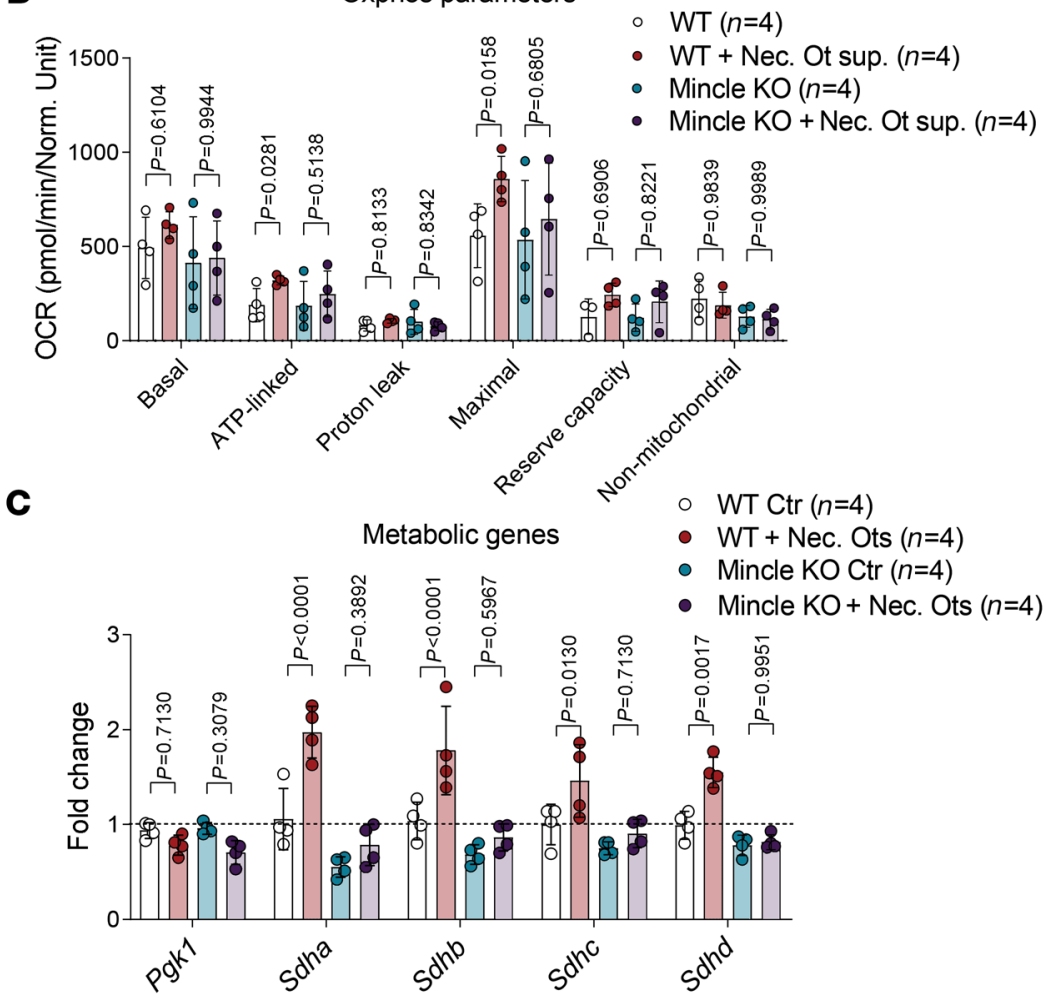

D
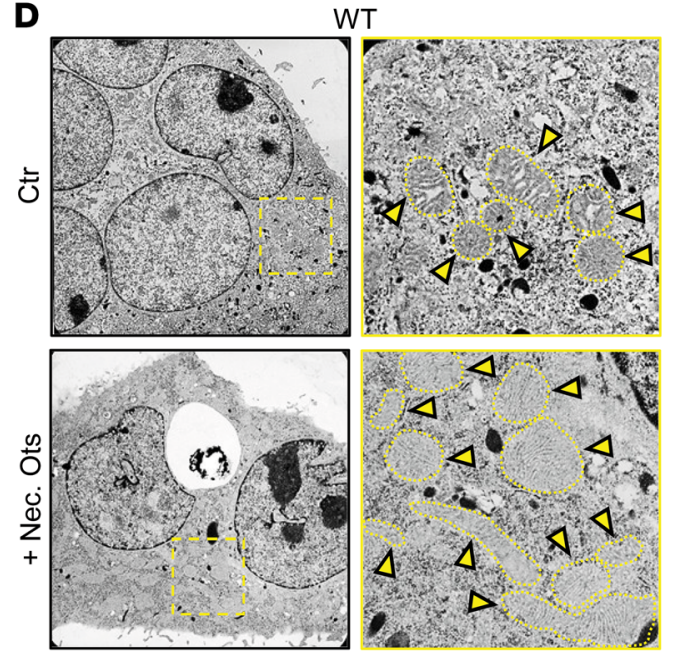

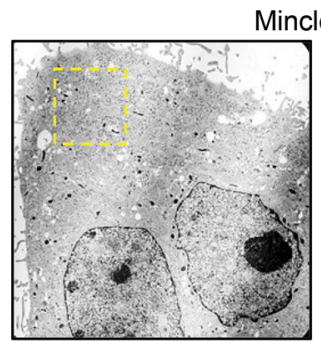

Mincle KO

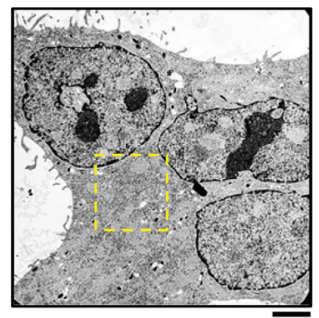

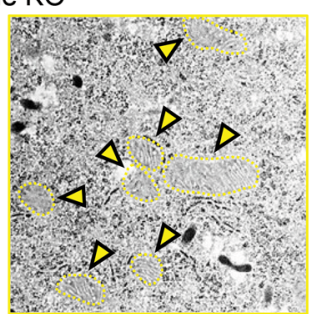

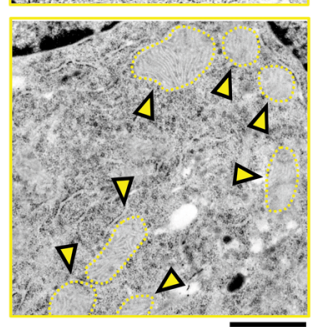

E

$\mathrm{WT}+\mathrm{Nec}$. Ots

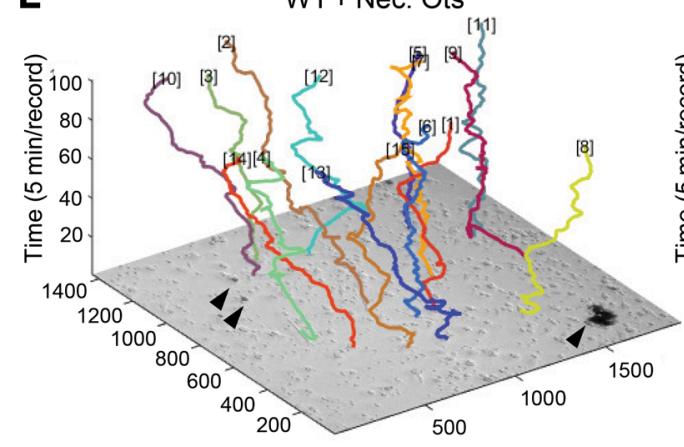

Mincle $\mathrm{KO}+\mathrm{Nec}$. Ots

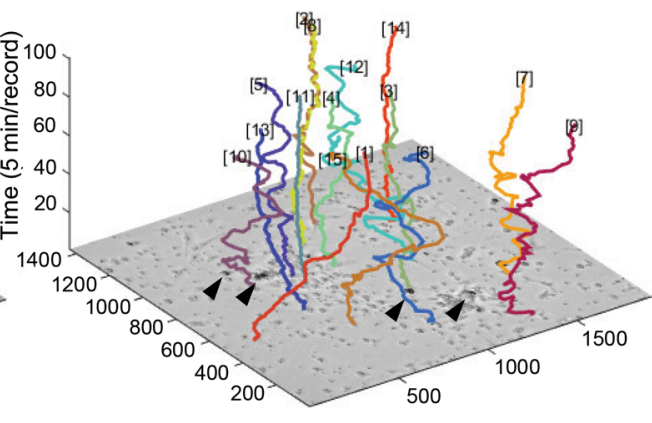

F Cell movement

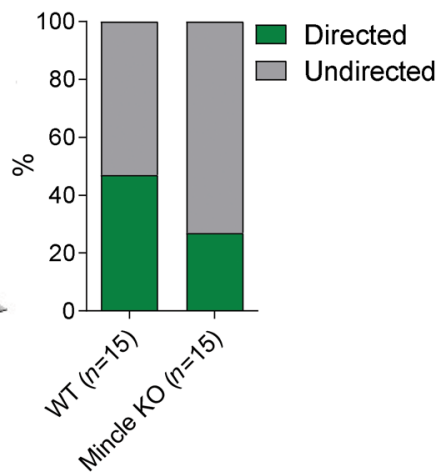


Figure 4. DAMP/Mincle axis skews metabolic activity of osteoclasts to oxidative phosphorylation and directs osteoclast migration. (A) Oxygen consumption rate (OCR) profile plot and (B) mitochondrial function parameters analyzed by extracellular flux assay in osteoclasts from WT and Mincle-KO mice, which were supplemented with necrotic osteocyte (0t) supernatant (1:2) for 24 hours on day 1 of culture, compared with nonsupplemented controls ( $n=4$ /group). A\&R, antimycin $A$ and rotenone. (C) Gene expression analysis of metabolic markers Pgk1, Sdha, Sdhb, Sdhc, and Sdhd in osteoclasts from WT and Mincle-KO mice, which were supplemented with necrotic osteocytes (1:2) for 24 hours on day 1 of culture, compared with controls ( $n=4 /$ group). (D) Transmission electron microscopy (TEM) images of WT and Mincle-KO osteoclasts, supplemented on day 1 for 24 hours with necrotic osteocytes (1:2), compared with nonsupplemented controls. Yellow arrows show mitochondrial cristae within the cells. Scale bars: $2.6 \mu \mathrm{m}$ (low-power images, left columns) and $1.21 \mu \mathrm{m}$ (high-power images, right columns). (E) 3D track plots of the recorded movement of WT and Mincle-KO osteoclasts within 500 minutes (recorded every 5 minutes) after stimulation with necrotic osteocytes. Black arrows show necrotic osteocytes. (F) Percentage of WT and Mincle-KO osteoclasts moving toward necrotic osteocytes in a directed way or moving without direction ( $n=15$ /group). Data are shown as mean $\pm \mathrm{SD}$. $P$ values were determined by 2-way ANOVA for multiple comparisons (B and $\mathbf{C}$; interaction $P$ value: 0.7410 for $\mathbf{B}$ and $<0.0001$ for $\mathbf{C}$ ).

TRAP-positive osteoclasts, covering a smaller area of woven bone within the fracture callus (Figure 6, G and H). These data suggest that targeting Mincle may inhibit inflammation-mediated systemic bone loss and enhance fracture repair.

Mincle deficiency mitigates osteocyte necrosis-induced bone loss. To investigate whether Mincle is essentially involved in osteoclast activation in the context of osteonecrosis, we analyzed CD $68^{+} \mathrm{Min}-$ $\mathrm{cle}^{+}$cells in the long bones of Dmp1-Cre ${ }^{+} / \mathrm{iDTR}^{\mathrm{f} / \mathrm{fl}}$ mice, exposed to DT for induction of osteocyte death. Indeed, the number of Mincle-positive osteoclasts was upregulated after DT injection in Dmp1-Cre ${ }^{+} / \mathrm{iDTR}^{\mathrm{f} / \mathrm{fl}}$, but not in Dmp1-Cre ${ }^{-} / \mathrm{iDTR}^{\mathrm{f} / \mathrm{fl}}$ controls (Figure 7, A and B). To test whether the absence of Mincle can prevent osteocyte death-induced bone loss, we generated Mincle-deficient Dmp1-Cre ${ }^{-} / \mathrm{iDTR}^{\mathrm{fl} / \mathrm{fl}}$ and Dmp1-Cre ${ }^{+} / \mathrm{iDTR}^{\mathrm{fl} / \mathrm{ll}}$ mice, which were compared with Mincle-competent Dmp1-Cre ${ }^{+} / \mathrm{iDTR}^{\mathrm{fl} / \mathrm{fl}}$ and Dmp1$\mathrm{Cre}^{-} / \mathrm{iDTR}^{\mathrm{f} / \mathrm{fl}}$ littermate controls upon DT injection. As shown in Figure 7, C-F, Mincle deletion hampered osteonecrosis-mediated bone loss and osteoclast induction. Likewise, in Dmp1-Cre ${ }^{+} / \mathrm{iDTR}^{\mathrm{f} / \mathrm{t}}$ mice that received hematopoietic stem cells from Mincle-KO mice (lacking Mincle on hematopoietic cells, but being sensitive to DT), DT administration was no longer able to trigger bone loss and increased osteoclast numbers (Supplemental Figure 11, A-D). These data show that Mincle is activated by necrotic osteocytes and is essential for bone loss after osteocyte death.

Mincle regulates human osteoclastogenesis and is upregulated in osteonecrotic bone lesions. To address whether our findings may also be relevant for human bone disease, mRNA expression of the human gene encoding Mincle (CLEC4E) was analyzed during the differentiation of human osteoclasts and compared with the expression of the osteoclast marker mRNAs NFATC1 and CTSK (Figure 8A). Similar to the observation in mice, CLEC4E was upregulated during the differentiation of human osteoclasts (Figure 8A). Additionally, the stimulation of human osteoclasts with the Mincle ligand TDB or necrotic cells also induced mRNA expression of CLEC4E, especially after 1 hour and 3 hours (Figure $8 \mathrm{~B}$ ) together with the osteoclast-related genes TNFRSF11A, NFATC1, ACP5, and MMP9 (Supplemental Figure 12A). Moreover, osteoclastogenesis was enhanced upon stimulation with TDB or necrotic cells (Figure 8, C-F). These results suggest that Mincle also promotes human osteoclast formation.

Because our murine data showed that Mincle-positive osteoclasts are upregulated in the context of osteonecrosis, we assessed Mincle expression in osteonecrotic bone from patients with 2 different diseases: osteonecrosis of the femoral head (ONFH) and medication-related osteonecrosis of the jaw (MRONJ), both characterized by profound osteocyte death and high osteoclast numbers compared with healthy bone sections (Figure 9, A and B). Immunofluorescence staining of Mincle and CD68 and DAPI staining revealed increased numbers of Mincle-positive osteoclasts in ONFH as well as in MRONJ bone lesions (Figure 9, C-E). Although the expression of Mincle did not correlate with earlyosteoclast markers TNFRSF11A, OSCAR, and NFATC1 (Supplemental Figure 12B), a positive correlation was observed between Mincle and osteoclast activity markers, such as cathepsin $\mathrm{K}$ and matrix metalloproteinase 9 in the osteonecrotic lesions (Figure 9F). These data provide evidence that Mincle may link osteocyte death and bone loss also in human bone disease.

\section{Discussion}

In this study, we identified a mechanistic link between osteocyte death and bone loss, which is mediated by osteocyte-derived DAMPs that are recognized by the C-type lectin receptor Mincle on osteoclasts. Mincle allows osteoclast lineage cells to respond to injury-induced osteocyte death, leading to increased osteoclast differentiation and bone resorption. Hence, inhibition of Mincle could be a new concept for specifically treating bone diseases associated with osteocyte death.

Enhanced osteocyte death is a key feature in many, but not all forms of bone diseases and is frequently observed in osteonecrosis, fracture, and inflammatory bone disease. It is considered that dying osteocytes stimulate osteoclast-mediated bone resorption, but the molecular mechanism of this link remained unclear to date. Cheung et al. showed that osteocyte apoptosis stimulates adjacent healthy osteocytes to secrete RANKL, which induces osteoclast differentiation (35). However, apoptosis of osteocytes is rapidly followed by secondary necrosis, because osteocytes are located in isolated lacunae within the bone matrix and the apoptotic cells cannot be engulfed by phagocytic cells. The necrotic osteocytes lose membrane integrity and release DAMPs into the extracellular space $(10,36)$. Here we show that Mincle-mediated sensing of DAMPs from necrotic osteocytes leads to robust osteoclast activation, explaining the wave of osteoclast activity in conjunction with osteocyte death.

Whole-transcriptome RNA-seq analyses showed that the DAMP/Mincle axis induces calcium signaling and oxidative phosphorylation in osteoclasts. Calcium signaling plays a crucial role in the differentiation and function of osteoclasts. The coreceptor/calcium signaling pathway in osteoclasts provides a RANKLindependent activation of NFATc1 (37). In addition, several publications shed light on the mechanistic link between calcium oscillation and mitochondrial expansion in osteoclasts $(38,39)$. Thus, our data show that Mincle engagement by osteonecrotic DAMPs substantially shifts the metabolic activity of osteoclasts toward 
A

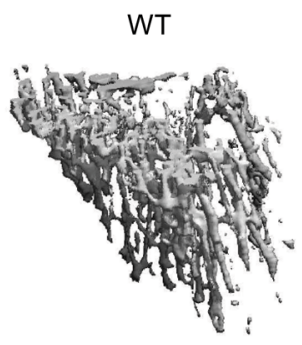

Mincle KO

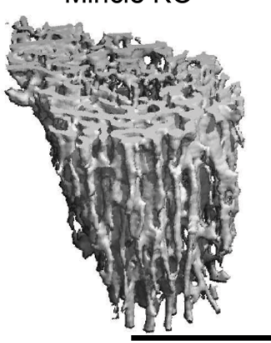

C

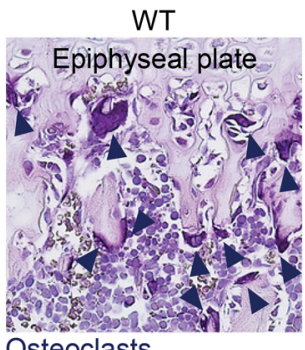

Osteoclasts

E

WT
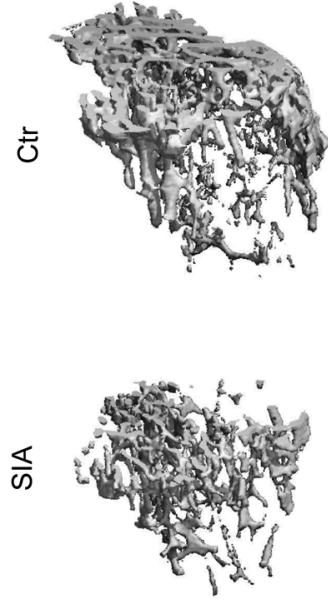

G WT

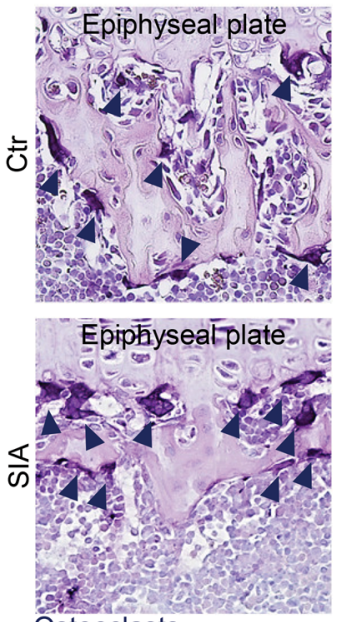

Mincle KO
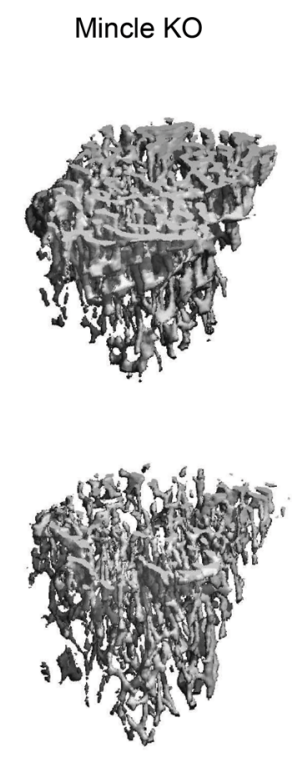

Mincle KO

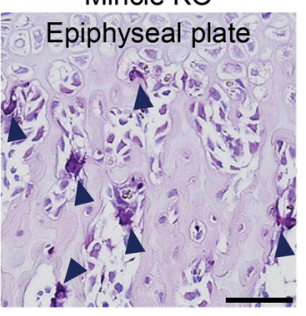

Mincle KO

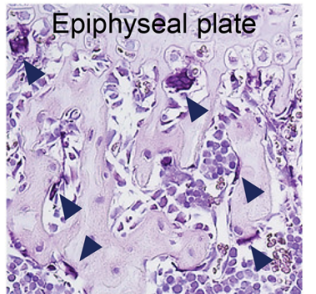

"Epiphysear plate:

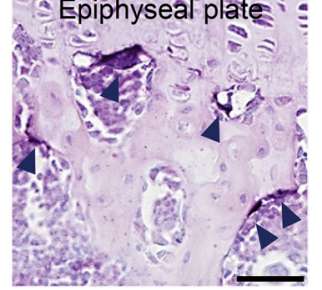

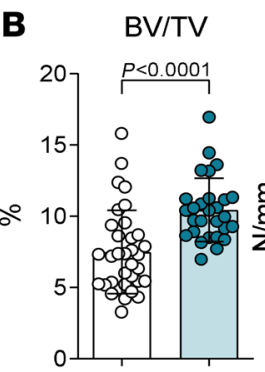
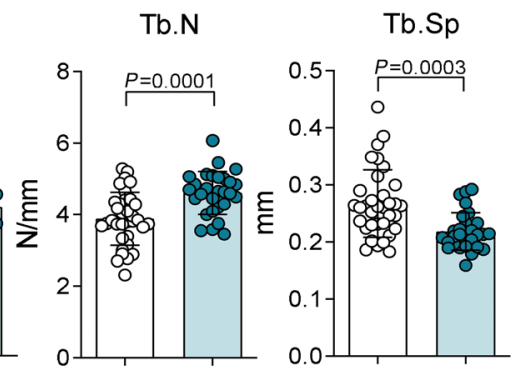

- WT $(n=34)$ - Mincle KO $(n=28)$

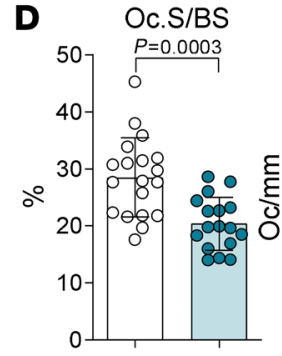

Oc.N/B.Pm

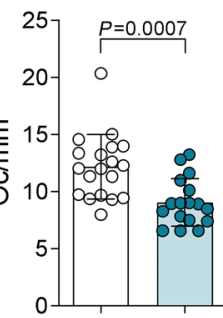

○ WT $(n=17)$

- Mincle KO $(n=15)$

\section{$\mathbf{F}$}
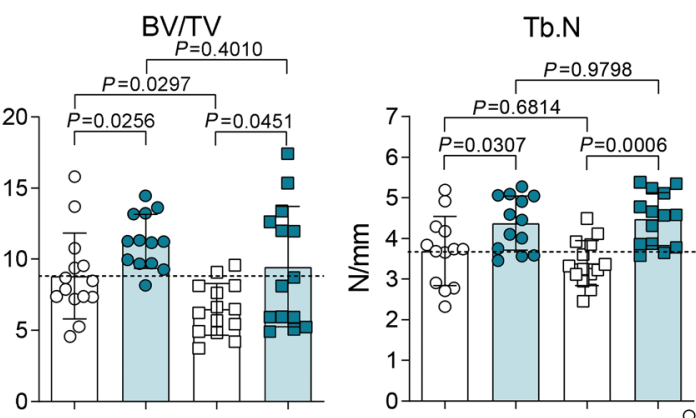

Tb.Sp

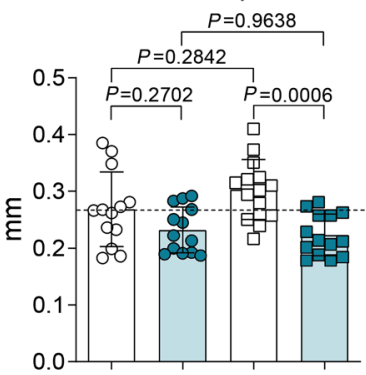

Tb.Th

- WT $(n=14)$

- Mincle KO $(n=13)$

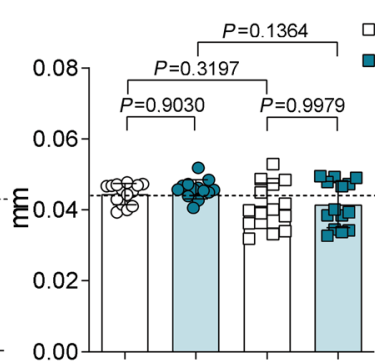

WT SIA $(n=14)$

Mincle KO SIA $(n=14)$

H

Oc.S/BS

Oc. N/B.Pm
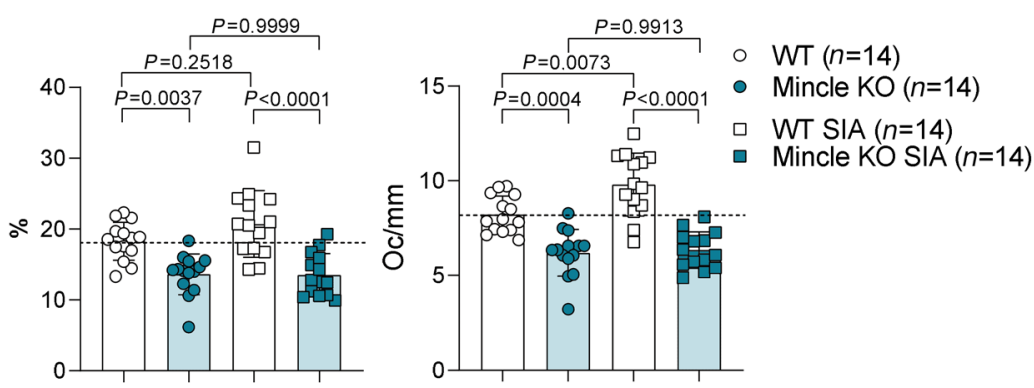
Figure 5. Mincle-KO mice exhibit high bone mass due to reduced osteoclast numbers and are protected from inflammation-mediated systemic bone loss. (A) Representative $\mu \mathrm{CT}$ images of tibial bones from 9-week-old WT controls and Mincle-KO mice and (B) quantification of BV/TV, Tb.N, Tb.Sp, and Tb.Th ( $n=28-34 /$ group). Scale bar: $1 \mathrm{~mm}$. (C) Representative images and (D) histomorphometric quantification of Oc.S/BS and Oc.N/B. $\mathrm{Pm}$ in TRAP-positive tibial sections of WT and Mincle-KO mice $(n=15-17)$ group). Dark blue arrows indicate the purple-stained osteoclasts. Scale bar: $50 \mu \mathrm{m}$. (E) Representative $\mu \mathrm{CT}$ images and (F) quantification of BV/ TV, Tb.N, Tb.Sp, and Tb.Th in tibial bones of 9-week-old WT and Mincle-KO mice, 9 days after serum-induced arthritis (SIA), compared with nonarthritic controls ( $n=13-14 /$ group). Scale bar: $1 \mathrm{~mm}$. (G) Representative TRAP staining of tibial sections from the aforementioned 4 groups. Dark blue arrows indicate the purple-stained osteoclasts. Scale bar: $50 \mu \mathrm{m}$. (H) Histomorphometric quantification of Oc.S/BS and Oc.N/B.Pm in tibial bones of the aforementioned 4 groups ( $n=14$ /group). Data are shown as mean \pm SD. $P$ values were determined by 2-tailed Student's $t$ test for single comparisons ( $\mathbf{B}$ and $\mathbf{D}$ ) or 1-way ANOVA for multiple comparisons ( $\mathbf{F}$ and $\mathbf{H}$ ).

oxidative phosphorylation. These observations are in accordance with previous studies showing that mitochondrial energy metabolism is required for osteoclastogenesis (38) and that mitochondrial dysfunction is linked to impaired osteoclast differentiation (40).

Mincle-mediated osteoclastogenesis appears to be particularly important in diseases associated with osteocyte death. Nonetheless, the observation that Mincle-KO mice exhibit increased bone mass at baseline indicates that some level of osteocyte death occurs continuously and influences bone resorption. Some studies have shown a higher proportion of dead osteocytes in postmenopausal osteoporotic bone in mice and humans $(41,42)$, most probably due to the loss of prosurvival functions of estrogen (43). However, it is still unclear whether osteocyte death is only an outcome or a possible cause of postmenopausal bone decline. Our study demonstrates that Mincle deficiency is not able to compensate for bone loss upon ovariectomy, although Mincle-KO mice show an overall higher bone mass and reduced osteoclast number, assuming that osteocyte death plays no crucial role during the establishment of bone loss in estrogen deficiency. The main consequence of estrogen withdrawal is an increased RANKL secretion, which constantly drives further bone resorption (44). It is therefore likely that Mincle depletion is not sufficient to counteract RANKLmediated bone loss, since RANKL acts via an independent receptor. Similar observations were seen in DAP12 ${ }^{--} \mathrm{FcR} \gamma^{-1-}$ mice that show lack of osteoclasts at baseline, but are still able to develop significant bone decline after ovariectomy (45).

A strong impulse for osteocyte death and DAMP release, however, occurs in fracture. In support of the concept that DAMPmediated Mincle activation triggers osteoclastogenesis, Mincle-KO mice exhibited reduced osteoclast numbers after fracture, which allows the formation of a denser callus, providing better stability. At the same time, Mincle-KO mice were still able to properly remodel the bony callus to functional secondary bone, meaning they normally completed the healing process. Thus, targeting Mincle may provide a therapeutic option to speed up fracture healing, especially in those with impaired repair. Along the same line, RA-induced inflammatory bone loss appears to be Mincle dependent, as Mincle-KO mice exhibited less pronounced bone loss despite higher inflammatory burden. The latter observation may be based on the previously reported ability of Mincle to skew macrophages toward an antiinflammatory phenotype (21). Remarkably, osteoclast activation and bone loss in long bones were clearly reduced, suggesting a strong impact of osteocyte death on osteoclast activation during inflammatory arthritis.

Osteocyte death is also a central factor in the pathogenesis of osteonecrosis, which is triggered by the disruption of blood supply to confined areas of the bone. Mincle-positive osteoclasts are upregulated in the 2 key manifestations of human osteonecrosis: MRONJ and ONFH $(46,47)$. Collapse of bone in osteonecrosis is caused by severe disturbance of bone remodeling with lack of bone formation, but increased bone resorption (48) with osteoclasts located in the necrotic bone region in rodents and humans (49-51). Accumulation of osteoclasts in ONFH likely depends on Mincle-sensing necrotic osteocytes. Interestingly, Mincle upregulation and its relation to osteoclast activity was also found in MRONJ. MRONJ primarily develops as a consequence of high-dose and long-term treatment with antiresorptive drugs that block osteoclast activity, arrest bone remodeling, and promote osteocyte death (46). Although some data suggested low numbers of osteoclasts in MRONJ $(52,53)$, others showed giant osteoclasts in the jaws of mice $(54,55)$ and humans (56). This discrepancy may be based on the stage of the lesion. During the development of necrosis, no or low osteoclasts may be found, while at later stages a wave of newly Mincle-induced osteoclasts may emerge as an attempt to remove dead bone.

In summary, our data identify the PRR Mincle as being responsible for osteoclast differentiation upon injury-induced bone damage, allowing the recognition of necrotic osteocytes. Mechanistically, Mincle is activated by DAMPs released from dying osteocytes and enhances osteoclastogenesis via ITAM-based induction of calcium signaling and oxidative phosphorylation. Thus, Mincle triggers increased osteoclastic activity in bone diseases associated with osteocyte death, including fracture, arthritis, and osteonecrosis. Therefore, inhibition of Mincle may offer a specific therapeutic intervention for uncoupling osteocyte death from osteoclast activation.

\section{Methods}

Mice. All mice used were on the C57BL/6 background. Mincle-KO mice were initially generated by the Consortium for Functional Glycomics (57) and described previously (20). Mincle-KO mice were crossed with C57BL/6 mice from Charles River. C57BL/6 WT littermates were used as controls in our experiments. Steady-state bone phenotype was analyzed in male, 9-week-old or 5-month-old animals. Dmp1-Cre mice were obtained from Jackson Laboratory [B6N.FVB-Tg (Dmp1-cre) 1Jqfe/BwdJ] (58) and crossed with iDTR mutant mice, also obtained

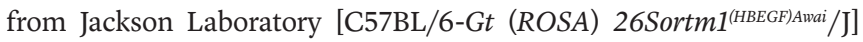
(59). In addition, Mincle-KO mice were crossed with Dmp1-Cre mice. All animals were maintained in a specific pathogen-free (SPF) facility with a temperature between $22^{\circ} \mathrm{C}$ and $23^{\circ} \mathrm{C}$, humidity of $50 \%-60 \%$, and a regulated 12-hour light/dark cycle. The animals were provided with normal diet food (ssniff, V1534-000) and water ad libitum.

Osteocyte ablation. Nine-week-old, female Dmp1-Cre ${ }^{-} / \mathrm{iDTR}^{\mathrm{fl} / \mathrm{fl}}$ littermate control, Dmp1-Cre ${ }^{+}$iDTR $^{\mathrm{fl} / \mathrm{fl}}$, Mincle-KO-crossed Dmp1$\mathrm{Cre}^{-} / \mathrm{iDTR}^{\mathrm{fl} / \mathrm{fl}}$, and Mincle-KO-crossed Dmp1-Cre ${ }^{+} / \mathrm{iDTR}^{\mathrm{fl} / \mathrm{ll}}$ mice were injected i.p. with $100 \mathrm{ng}$ DT (MilliporeSigma) dissolved in PBS. Mice were analyzed 4 days after DT injection.

$K / B x N$ SIA. K/BxN SIA was induced in 8-week-old, male WT control and Mincle-KO mice by i.p. injection of $150 \mu \mathrm{L}$ pooled serum from 
A

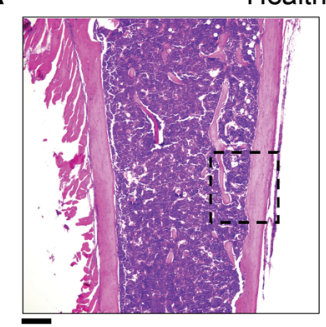

Healthy bone

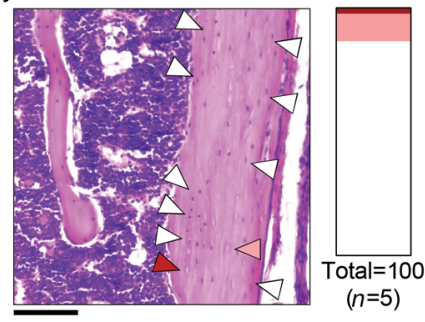

$\square 2.4 \%$ Empty lacunae

$\square 11.3 \%$ Dying osteocytes
$\square 86.3 \%$ Filled lacunae
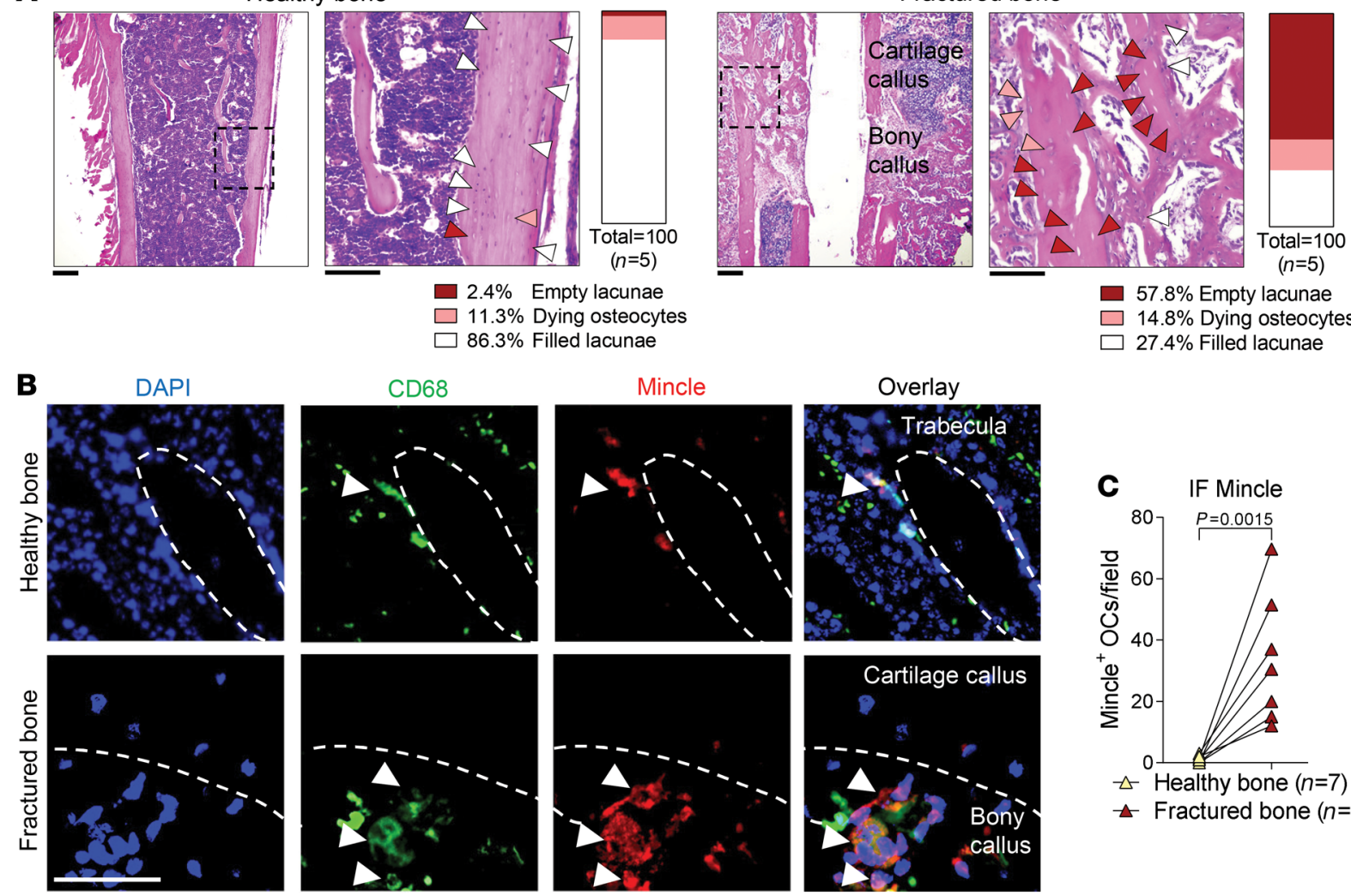

$\square 57.8 \%$ Empty lacunae

$\square 14.8 \%$ Dying osteocytes

$\square 27.4 \%$ Filled lacunae
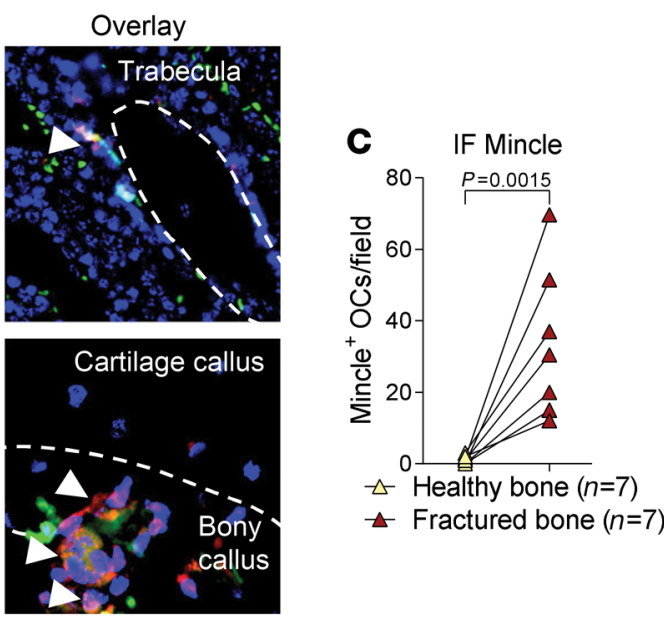
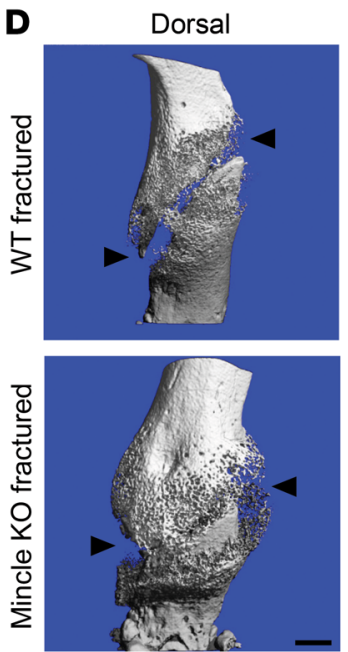

G

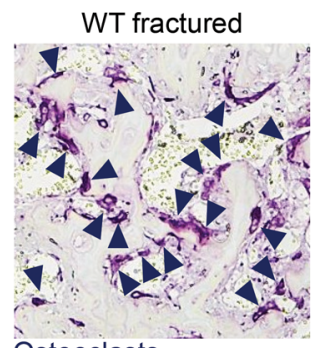

Thickness
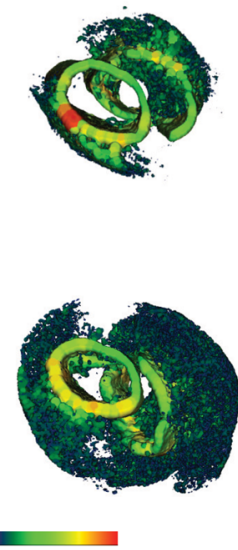

Mincle KO fractured

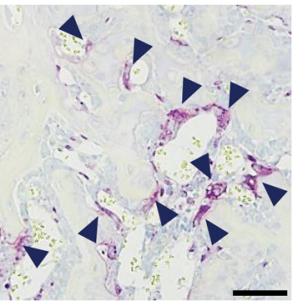

E
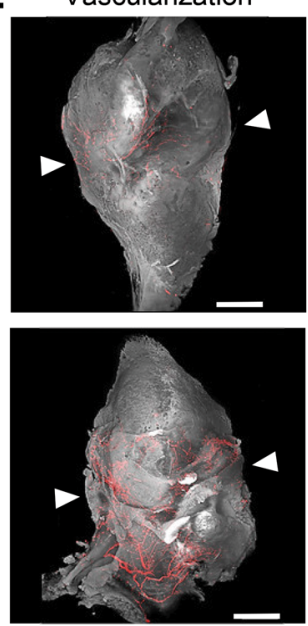

\section{F Callus BV $/ \mathrm{TV}$}

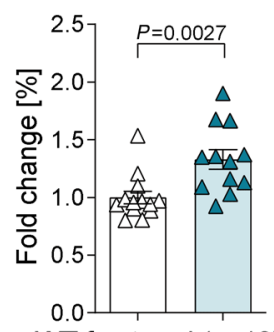

$\triangle$ WT fractured $(n=13)$

$\Delta$ Mincle KO fractured $(n=12)$
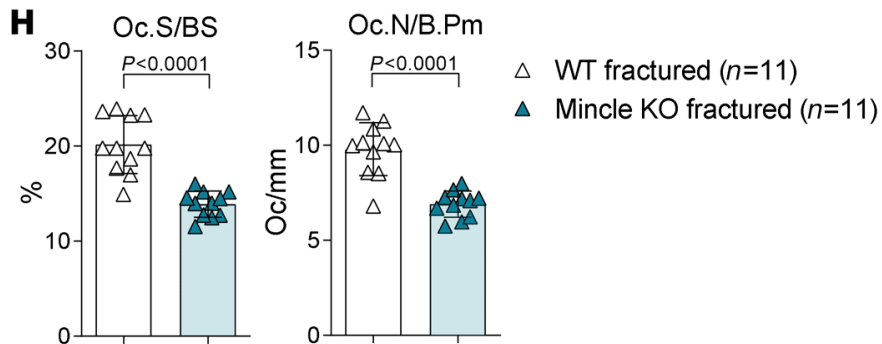
Figure 6. Mincle-KO mice show improved fracture healing. (A) Representative pictures of H\&E staining of femoral bone 14 days after fracture compared with healthy tibial bone at 14 weeks of age. Scale bars: $200 \mu \mathrm{m}$ (left) and $100 \mu \mathrm{m}$ (right). Quantification of filled lacunae (white arrows), dying osteocytes (orange arrows), and empty lacunae (red arrows) ( $n=5 /$ group). (B) Immunofluorescence (IF) staining for CD68 (green) and Mincle (red) and DAPI staining (blue) in unaffected tibial bones compared with fractured femoral bones. White arrows indicate Mincle-positive osteoclasts. Scale bar: $50 \mu \mathrm{m}$. (C) Quantification of Mincle-positive osteoclasts (OCs) ( $n=7 /$ group). (D) Representative $\mu \mathrm{CT}$ images of femoral bones of WT and Mincle-KO mice, 14 days after fracture at 14 weeks of age, showing the dorsal view and the thickness of the fracture callus. Black arrows show the fracture gap. Scale bar: $1 \mathrm{~mm}$. (E) Light-sheet fluorescence microscopy (LSFM) of vascularization (CD31, red) in the callus (autofluorescence, gray) of the aforementioned 2 groups. Scale bar: $1 \mathrm{~mm}$. (F) Quantification of BV/ TV in the fracture callus of the aforementioned 2 groups ( $n=12-13$ /group). (G) Representative TRAP staining in the bone callus of WT and Mincle-KO mice. Dark blue arrows indicate the purple-stained osteoclasts. Scale bar: $50 \mu \mathrm{m}$. (H) Histomorphometric quantification of Oc.S/BS and Oc.N/B.Pm in the hard callus of Mincle-KO compared with WT mice ( $n=11 /$ group). Data are shown as mean \pm SD. $P$ values were determined by 2-tailed Student's $t$ test for single comparisons (C, $\mathbf{F}$, and $\mathbf{H})$.

arthritic adult K/BxN mice, as described previously (33). Development of arthritis was evaluated in each paw, using a semiquantitative scoring system (0-4 per paw; maximum score of 16) as previously described (60). In addition, weight loss was monitored over the course of the experiment. Paw and ankle swelling was measured with a digital caliper. The experiment ended 9 days after serum transfer at the age of 9 weeks.

Three-point-bending femur fracture. Twelve-week-old, female WT control or Mincle-KO mice were used for the fracture experiments. After anesthesia, using a ketamine hydrochloride/xylazine mixture (100 and $8 \mathrm{mg} / \mathrm{kg}$ body weight, i.p.), the right femur was fractured with 3-point bending (61). It was stabilized with an intramedullary nail (hollow needle, $24 \mathrm{G}$ ). Buprenorphine $(0.1 \mathrm{mg} / \mathrm{kg}$ body weight, s.c.) was given as an analgesic before and a second time after surgery. Additionally, buprenorphine $(10 \mu \mathrm{g} / \mathrm{mL})$ was given via the drinking water for 48 hours after fracture. The mice were analyzed 14 days, 21 days, or 3 months after surgery at the age of 14 weeks, 15 weeks, or 6 months, respectively.

Histological analysis. Long bones were fixed overnight in $4 \%$ paraformaldehyde (PFA) and afterwards decalcified in 14\% EDTA until bones were pliable. Serial paraffin sections $(2 \mu \mathrm{m})$ were stained with toluidine blue for quantifying osteoblasts and osteocytes and for TRAP, using a leukocyte acid phosphatase staining kit (MilliporeSigma) for detection of osteoclasts. Histomorphometric analysis of bone formation in tibias after calcein (MilliporeSigma) incorporation was performed on methacrylate-embedded undecalcified plastic sections. The quantification of osteoblasts (osteoblast surface per bone surface [Ob.S/BS], osteoblast number per bone perimeter [Ob.N/B.Pm]), osteocytes (osteocyte surface per bone surface [Ot.S/BS], osteocyte number per bone perimeter [Ot.N/B.Pm]), and osteoclasts (osteoclast surface per bone surface [Oc.S/BS], osteoclast number per bone perimeter [Oc.N/B.Pm]), as well as the bone formation rate per bone surface (mineral apposition rate [MAR], BFR/BS) was performed with a Zeiss Axioskop 2 microscope (Carl Zeiss), equipped with a digital camera and OsteoMeasure image analysis system (Osteometrics).

$\mu C T$. Long bones were fixed in $4 \%$ PFA overnight before the analyses. All $\mu \mathrm{CT}$ imaging was performed using the cone-beam
Desktop Micro Computer Tomograph $\mu$ CT 40 (SCANCO Medical). The settings were optimized for calcified tissue visualization at 55 $\mathrm{kVp}$ with a current of $145 \mu \mathrm{A}$ and $200 \mathrm{~ms}$ integration time for 500 projections $/ 180^{\circ}$. For the segmentation of $3 \mathrm{D}$ volumes, an isotropic voxel size of $8.4 \mu \mathrm{m}$ and an evaluation script with adjusted grayscale thresholds (threshold in the white range $>100$ ) of the operating system Open VMS (SCANCO Medical) was used. The analysis of the bone structure was performed in the proximal metaphysis of the tibia, starting $0.43-0.42 \mathrm{~mm}$ from an anatomic landmark in the growth plate and extending $1.68 \mathrm{~mm}$ (200 tomograms) distally. To determine the bone structure of the fractured femur callus, the center of the callus was identified and the bone volume (BV) was measured in this region, covering $2.10 \mathrm{~mm}$ (250 tomograms).

Immunofluorescence in bone sections. Epitopes were retrieved from deparaffinized sections by incubation in boiled Tris-EDTA buffer for 10 minutes. After washing with distilled water, sections were blocked with Streptavidin/Biotin blocking solution, according to the manufacturer's instructions (Invitrogen). In addition, the sections were blocked for 1 hour at room temperature in HEPES solution, supplemented with $2.5 \%$ horse serum (Vector Laboratories). Sections were incubated with primary antibodies for Mincle/CLEC4E (rabbit, Bioss, bs-8541R; 1:100) and CD68 (biotin, Genetex, GTX43914; 1:100) in HEPES solution, supplemented with $2.5 \%$ horse serum overnight at $4^{\circ} \mathrm{C}$. After washing, sections were incubated with the secondary antibody DyLight 594 Horse Anti-Rabbit IgG (Vector laboratories, DI-1094; 1:200) and Streptavidin-FITC (Southern Biotech, 7100-02; 1:50) in HEPES solution for 1 hour at room temperature and afterwards washed with PBS. Sections were mounted with fresh DAPI (MilliporeSigma) and covered with coverslips. Images were acquired at $\times 20$ magnification with the BZ-X700 All-in-One fluorescence microscope (Keyence). Quantification of Mincle-positive osteoclasts was performed with ImageJ (NIH), using Mincle-KO sections as a negative control.

Light-sheet fluorescence microscopy. For vascularization analyses of the fractured calli, WT and Mincle-KO mice were injected with 10 $\mu \mathrm{g} /$ mouse monoclonal anti-CD31 (BioLegend, 102502) labeled with Alexa Fluor 790 (Invitrogen), 14 days after fracture, 1 hour before ending the experiment. After sacrifice, the mice were perfused with PBS with 5 mM EDTA through the left ventricle and afterwards with $4 \%$ PFA to rinse erythrocytes and fix the bone tissue from inside, respectively. After fixing the femurs for an additional 4 hours with $4 \%$ PFA at $4^{\circ} \mathrm{C}$, the bones were dehydrated with increasing alcohol concentrations and cleared with ethyl cinnamate (MilliporeSigma), as described previously (62). The light-sheet fluorescence microscopy imaging was performed with a LaVision BioTec Ultramicroscope with an Olympus MVX10 zoom microscope body, a LaVision BioTec Laser Module, an Andor Neo sCMOS Camera with a pixel size of $6.5 \mu \mathrm{m}$, and detection optics with an optical magnification range of 1.263-12.63 and a numerical aperture (NA) of 0.5. To show the morphology of the callus, a $488 \mathrm{~nm}$ optically pumped semiconductor laser (OPSL) was used for generation of autofluorescent signals. For CD31-Alexa Fluor 790 excitation, we used a 785 nm OPSL. Emitted wavelengths were detected with specific detection filters: $525 / 50 \mathrm{~nm}$ for autofluorescence and 845/55 for CD31-Alexa Fluor 790. The optical zoom factor for measurements ranged from 1.0 to 1.6. Evaluations were done with ImageJ, using the 3Dscript (63).

IDG-SW3 osteocytic cell line. For osteocyte studies, we used the cell line IDG-SW3, which was provided by Erwin F. Wagner (Department of 
A
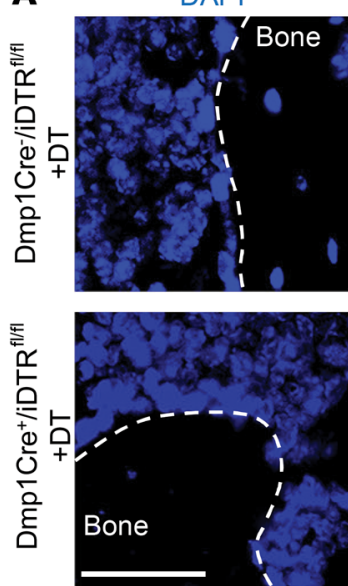

C Dmp1Cre-liDTR ${ }^{\text {fiff }}$

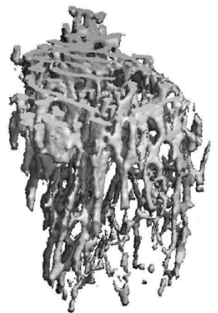

Mincle KO X Dmp1Cre-/iDTR ${ }^{\mathrm{fl} / \mathrm{fl}}$

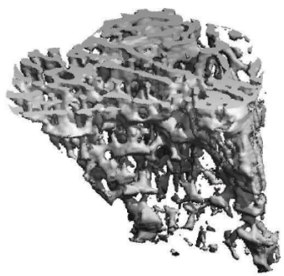

E

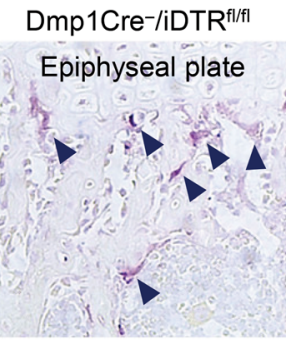

Mincle KOX Dmp1Cre-/iDTR R/fll Epiphyseal plate

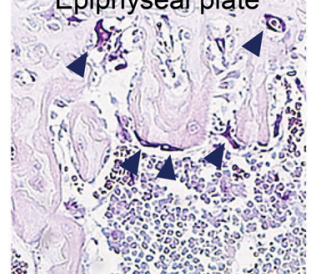

Osteoclasts
CD68
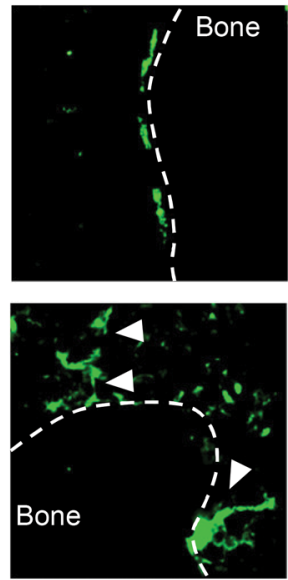

Dmp1Cre ${ }^{+} / \mathrm{iDTR}^{\mathrm{fl} / \mathrm{fl}}$

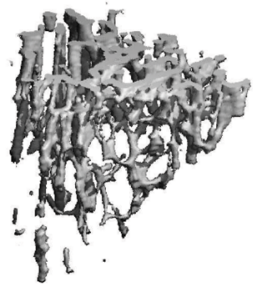

Mincle KOX Dmp1Cre $/ / \mathrm{iDTR}^{\mathrm{fl} / \mathrm{fl}}$

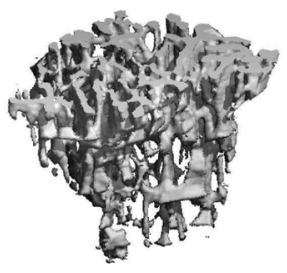

Dmp1Cre ${ }^{+} / \mathrm{iDTR}^{\mathrm{fl} / \mathrm{fl}}$ Ephiphyseal piate

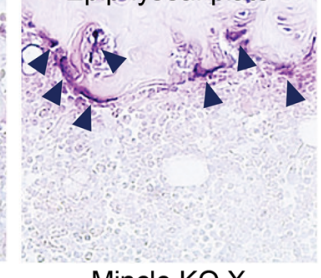

Mincle KO X Dmp1Cre $/$ iDTR $^{\mathrm{fl} / \mathrm{fl}}$ Epiphyseal plate

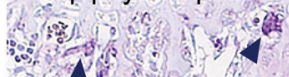
$=v_{6}$

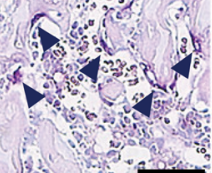

Mincle
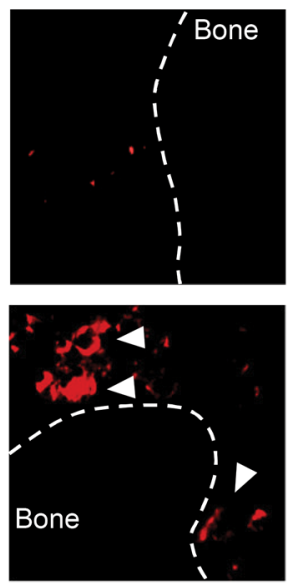

Overlay
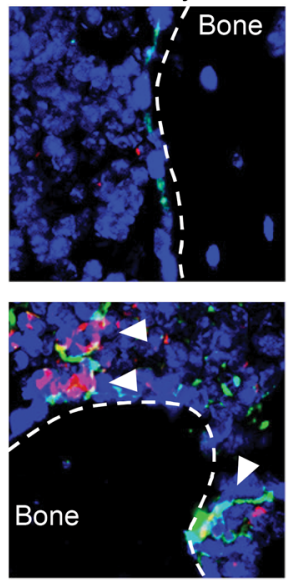

B

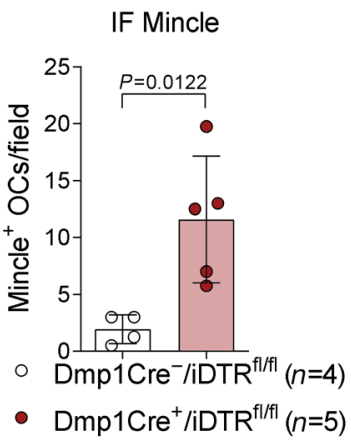

D

$\mathrm{BV} / \mathrm{TV}$

Tb.N

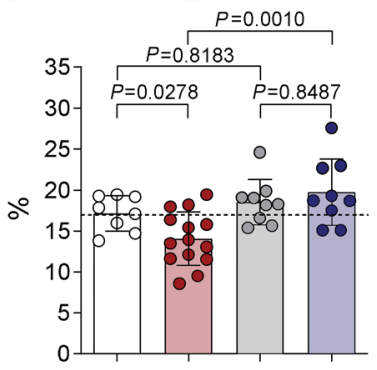

Tb.Sp

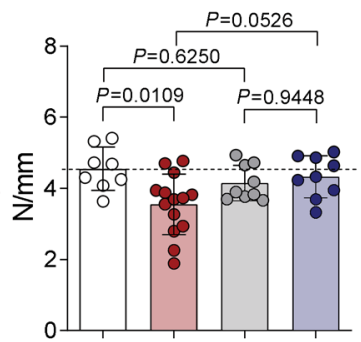

Tb.Th
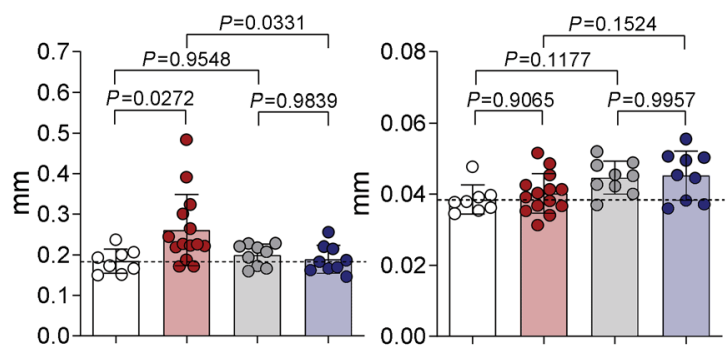

- Dmp1Cre $/ \mathrm{iDTR}^{\mathrm{f} / \mathrm{fl}}(n=8) \quad \circ \quad$ Mincle KO

X Dmp1Cre $/ \mathrm{iDTR}^{\mathrm{fl} / f l}(n=9)$

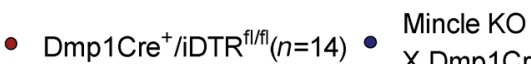

X Dmp1Cre ${ }^{+} / \mathrm{iDTR}^{f / / f l}(n=9)$

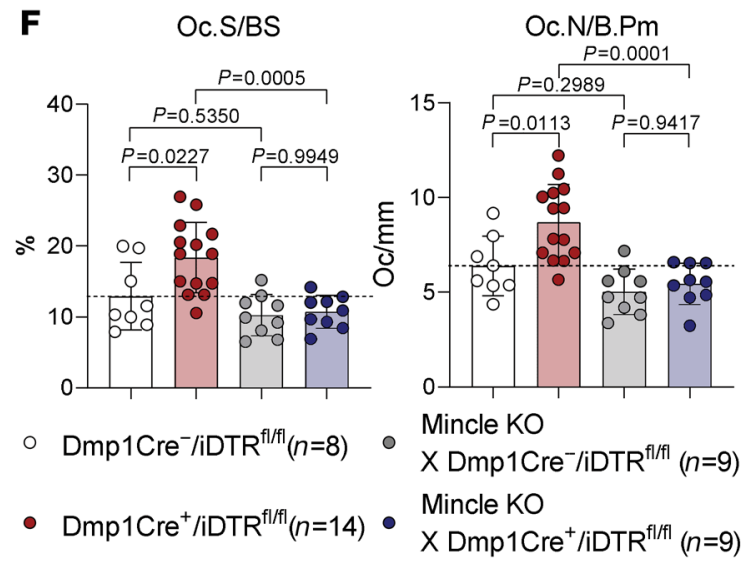


Figure 7. Mincle signaling is required for bone resorption upon osteocyte death. (A) Immunofluorescence (IF) microscopy of CD68 (green) and Mincle (red) and DAPI staining (blue), and (B) quantification of Mincle-positive osteoclasts in tibial bones of 9-week-old Dmp1-Cre $/ \mathrm{iDTR}^{\mathrm{fl} / \mathrm{fl}}$ mice compared with

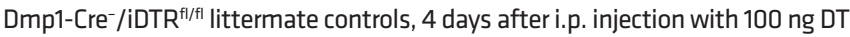
( $n=4-5 /$ group). White arrows show CD68 ${ }^{+}$Mincle $^{+}$cells near the bone surface. Scale bar: $50 \mu \mathrm{m}$. (C) Representative $\mu \mathrm{CT}$ images of tibial bones of 9-week-old Mincle-deficient Dmp1-Cre-/iDTR ${ }^{\mathrm{fl} / \mathrm{fl}}$ and Dmp1-Cre $/ \mathrm{iDTR}^{\mathrm{fl} / \mathrm{fl}}$ mice, compared with Mincle-competent Dmp1-Cre-/iDTR ${ }^{\mathrm{fl} / \mathrm{fl}}$ littermate controls and Dmp1-Cre ${ }^{+}$/ $\mathrm{iDTR}^{\mathrm{fl} / \mathrm{fl}}$ mice, 4 days after i.p. injection with $100 \mathrm{ng} \mathrm{DT}$ and (D) quantification of BV/TV, Tb.N, Tb.Sp, and Tb.Th ( $n=8-14 /$ group). Scale bar: 1 mm. (E) Representative TRAP staining of tibial bone sections from the 4 aforementioned groups. Dark blue arrows indicate purple-stained osteoclasts. Scale bar: $50 \mu \mathrm{m}$. (F) Histomorphometric quantification of Oc.S/BS and Oc.N/B.Pm in tibial bone from the 4 aforementioned groups ( $n=8-14 /$ group). Data are shown as mean \pm SD. $P$ values were determined by 2 -tailed Student's $t$ test for single comparisons (B) or 1-way ANOVA for multiple comparisons ( $\mathbf{D}$ and $\mathbf{F}$ ).

Dermatology and Department of Laboratory Medicine, MedUni Wien, Vienna). This cell line mimics osteoblast-to-late osteocyte differentiation in vitro. We differentiated IDG-SW3 cells at a density of $4 \times 10^{4}$ cells $/ \mathrm{cm}^{2}$ for 21 days to late osteocytes as previously described (64). To demonstrate osteocyte differentiation, the IDG-SW3 cell line expresses GFP under the DMP1 promoter. The GFP signal was controlled on day 21 of differentiation by comparison with IDG-SW3 cells on day 0 using the Keyence fluorescence microscope at $\times 20$ magnification. Necrotic osteocyte lysates were prepared by freezing the cells in liquid nitrogen and thawing them at room temperature. This cycle was repeated 4 times in total. Osteocyte necrosis was also induced by serum starvation (-FCS) for 24 hours, while the control cells received medium containing FCS (Biochrom). After 24 hours, the medium was exchanged again with FCS-containing medium in all conditions and collected after 24 hours for osteoclast stimulation. To demonstrate the successful induction of necrosis after 24-hour serum starvation, the osteocytes were detached from the plate with Accutase (Thermo Fisher Scientific) and stained with a mix $\left(500 \mu \mathrm{L}, 2 \times 10^{5}\right.$ cells) of $5 \mu \mathrm{g} / \mathrm{mL}$ propidium iodide (PI) (Amersham Biosciences) and $2.5 \mu \mathrm{g} / \mathrm{mL}$ Hoechst 33342 (Invitrogen) for 30 minutes at $4^{\circ} \mathrm{C}$ in the dark. After washing, this cell mixture was added to 8-well glass-bottom plates (Ibidi). The fluorescence signal of DMP1-GFP, PI, and Hoechst 33342 was measured with the Keyence fluorescence microscope and images were taken at $\times 20$ magnification. For live cell imaging, IDG-SW3 cells were cultured in 6-well plates and placed in the Keyence
A

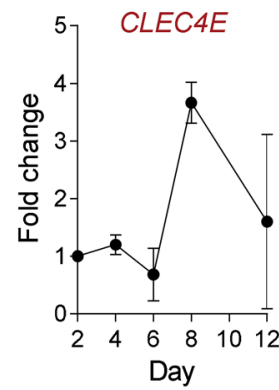

C

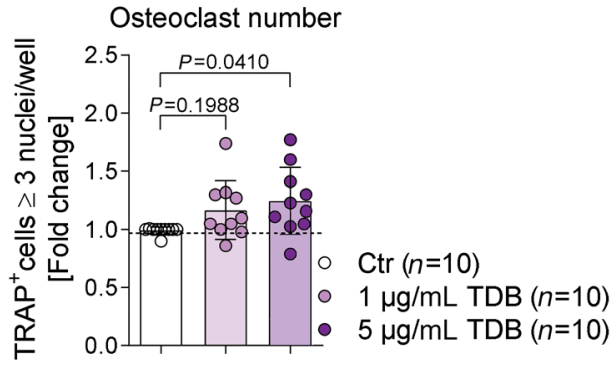

E

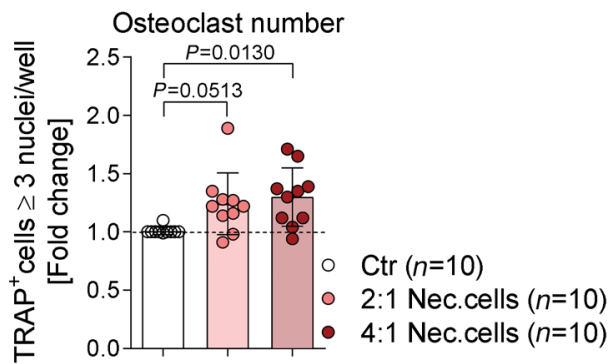

B

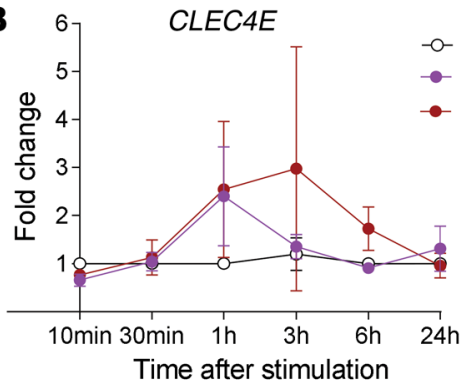

D

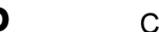

Ctr

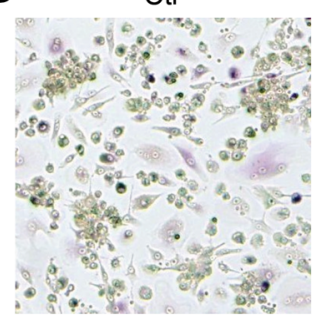

$1 \mu \mathrm{g} / \mathrm{mL}$ TDB
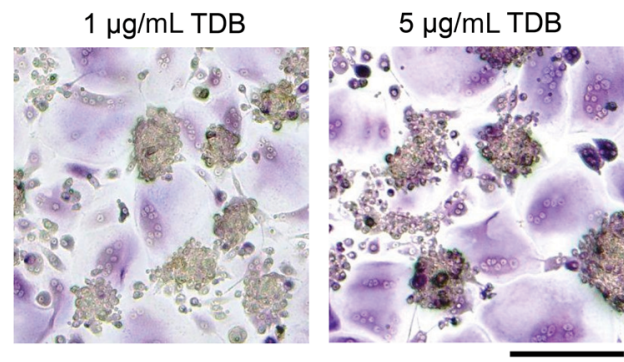

$\mathbf{F}$

Ctr

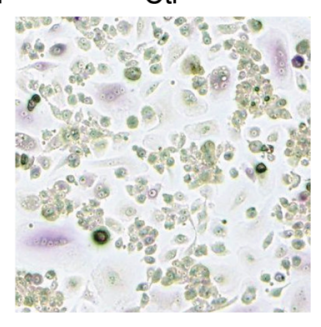

2:1 Necrotic cells

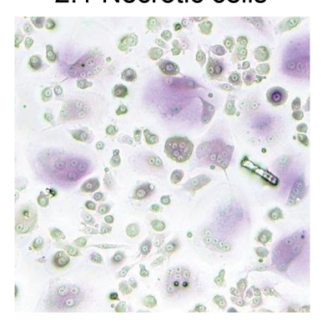

- C Ctr $(n=4)$

4:1 Nec. cells $(n=4)$
- $5 \mu \mathrm{g} / \mathrm{mL}$ TDB $(n=4)$

Figure 8. Mincle regulates human osteoclastogenesis. (A) Gene expression analysis of CLEC4E and the osteoclast markers NFATC1 and CTSK in osteoclasts, differentiated from human monocytes and analyzed on days 2, 4, 6, 8, and 12 of osteoclastogenesis. (B) Cene expression analysis of CLEC4E in human osteoclasts (day 5 of culture) 10 minutes, 30 minutes, 1 hour, 3 hours, 6 hours, and 24 hours after stimulation with $5 \mu \mathrm{g} / \mathrm{mL}$ TDB or with necrotic cells (PBMCs; 4:1 ratio), compared with a nonstimulated control ( $n=4 /$ group). (C) Quantification and (D) representative pictures of TRAP-positive human polynucleated ( $\geq 3$ nuclei) osteoclasts, supplemented with $1 \mu \mathrm{g} / \mathrm{mL}$ or $5 \mu \mathrm{g} / \mathrm{mL}$ TDB for 24 hours on day 5 of culture, compared with a nonsupplemented control ( $n=10$ /group). Scale bar: $50 \mu \mathrm{m}$. (E) Quantification and (F) representative pictures of TRAP-positive human polynucleated ( $\geq 3$ nuclei) osteoclasts, supplemented with 2:1 or 4:1 necrotic PBMCs for 24 hours on day 5 of culture, compared with a nonsupplemented control ( $n=10 / g r o u p)$. Scale bar: $50 \mu \mathrm{m}$. Data are shown as mean \pm SD. $P$ values were determined by 1-way ANOVA for multiple comparisons (C and $\mathbf{E})$. 
A
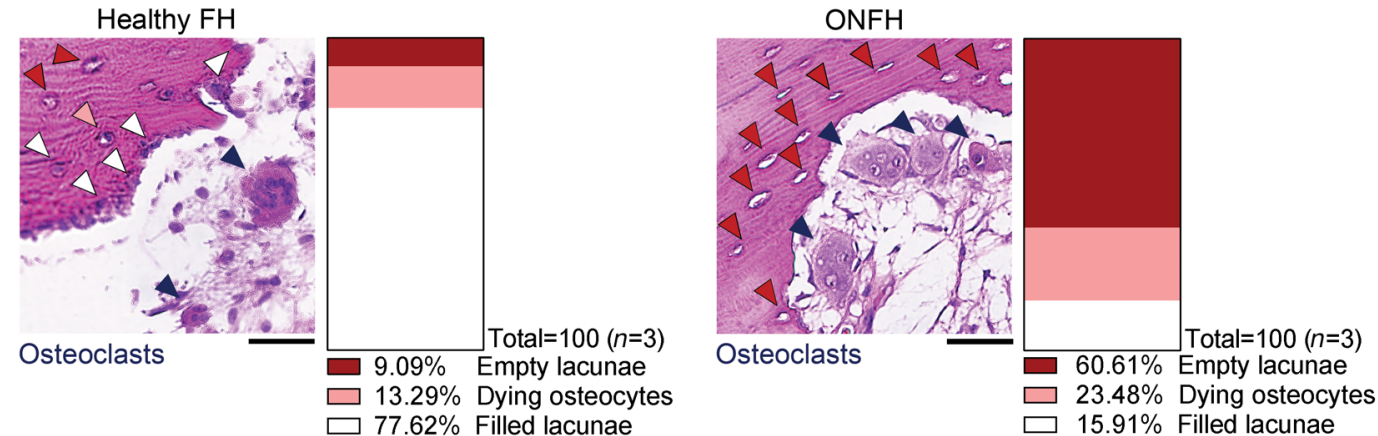

B
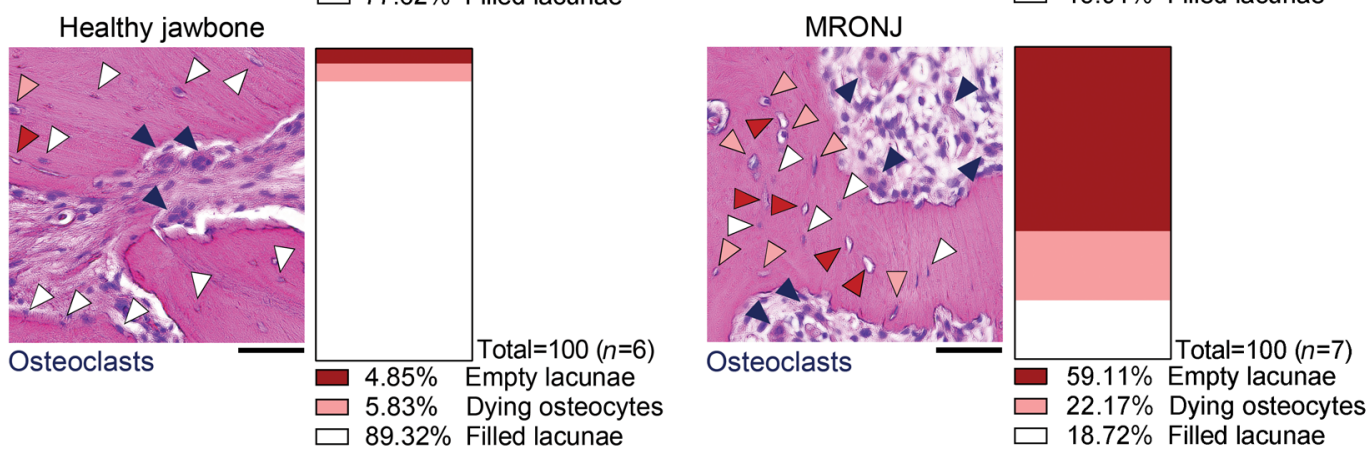

C
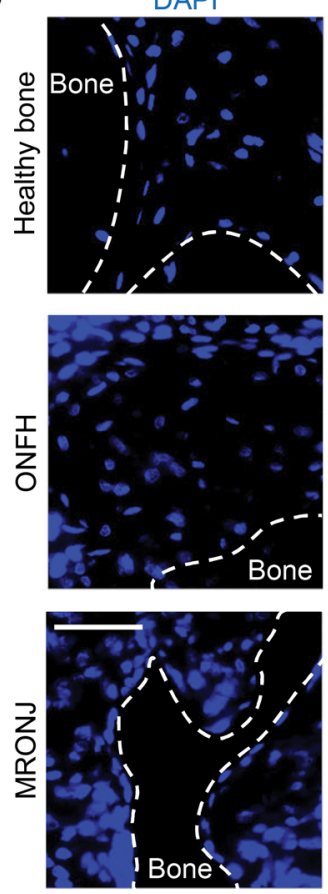

CD68
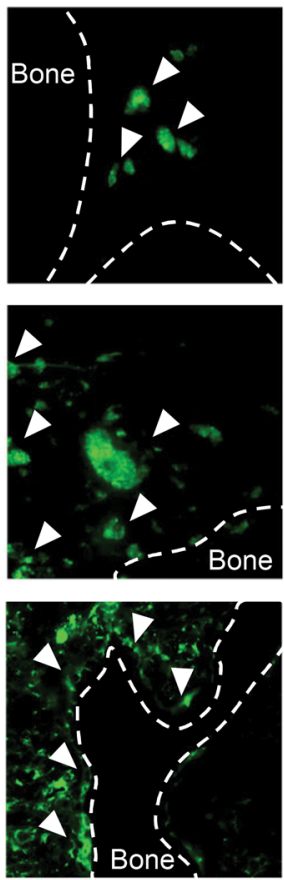

Mincle
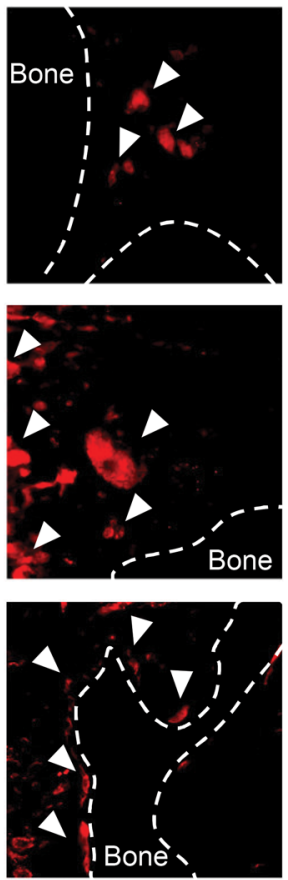

Overlay
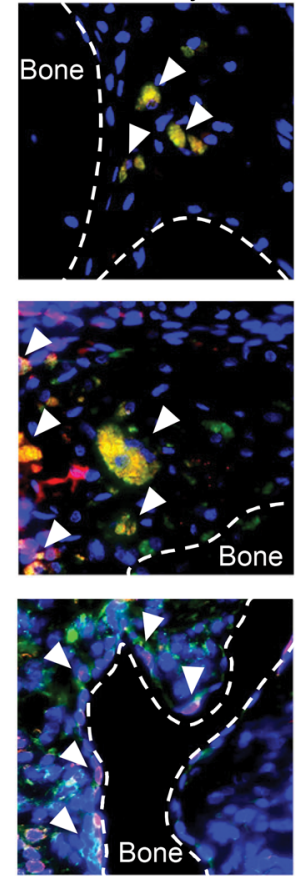

D

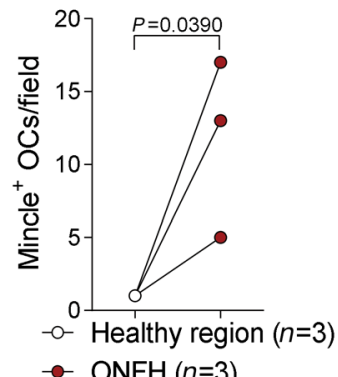

E

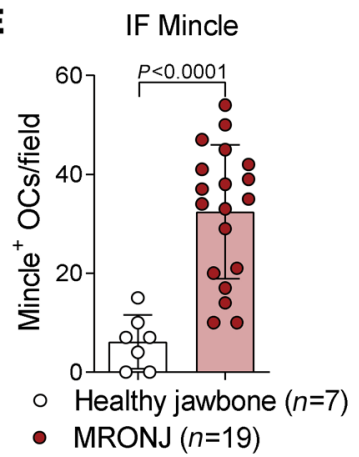

$\mathbf{F}$

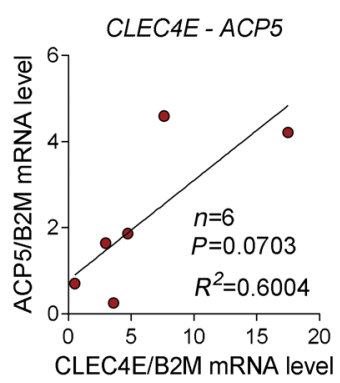

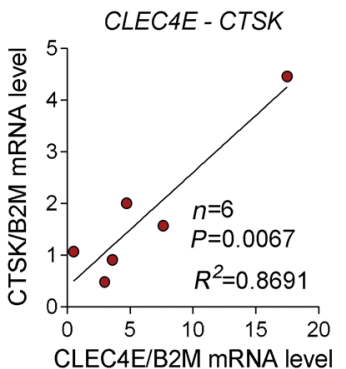

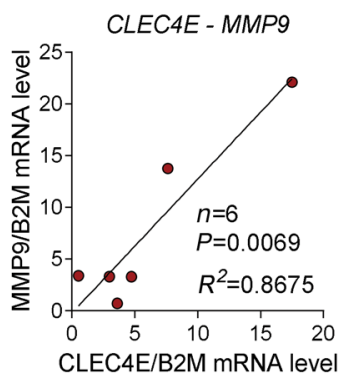


Figure 9. Mincle-positive osteoclasts are upregulated in osteonecrotic human bone lesions. Representative pictures of H\&E staining and quantification of filled lacunae (white arrows), dying osteocytes (orange arrows), and empty lacunae (red arrows) in (A) osteonecrosis of the femoral head (ONFH) lesions ( $n=3 /$ group) and (B) medication-related osteonecrosis of the jaw (MRONJ) lesions ( $n=6-7 /$ group), compared with the corresponding healthy bone areas. Dark blue arrows indicate polynucleated osteoclasts. Scale bar: $50 \mu \mathrm{m}$. (C) Immunofluorescence (IF) staining of CD68 (green) and Mincle (red) and DAPI staining (blue) (white arrows show CD68 ${ }^{+}$Mincle ${ }^{+}$ cells near the bone surface) in ONFH and MRONJ, compared with healthy regions. Scale bar: $50 \mu \mathrm{m}$. (D) Quantification of Mincle-positive osteoclasts (OCs) per field in ONFH, compared with healthy region ( $n=3$ /group). (E) Quantification of Mincle-positive osteoclasts per field in MRONJ, compared with healthy jawbone ( $n=7-19 /$ group). (F) Correlation analyses between the mRNA expression of CLEC4E and the osteoclast markers ACP5, CTSK, and MMPg in MRONJ samples $(n=6)$. Data are shown as mean \pm SD. $P$ values were determined by 2-tailed Student's $t$ test for single comparisons ( $\mathbf{D}$ and $\mathbf{E})$ and correlations were tested with the linear regression $F$ test $(\mathbf{F})$.

incubation chamber with or without FCS supplementation at $37^{\circ} \mathrm{C}$ and $5.5 \% \mathrm{CO}_{2}$. Shortly before starting the recordings, $5 \mu \mathrm{g} / \mathrm{mL}$ PI, $2.5 \mu \mathrm{g} /$ $\mathrm{mL}$ Hoechst 33342, and $20 \mathrm{nM} \mathrm{DilC}_{1}$ (5) (Invitrogen) were added to the cultures. Cells were monitored over 24 hours by taking images with the Keyence fluorescence microscope in preselected spots. Afterwards, the $Z$-stack images were stitched together and processed with ImageJ.

Measurement of SAP-130 levels. SAP-130 levels were measured by ELISA in the cell lysates of $10 \times 10^{6} / \mathrm{mL}$ splenocytes or IDG-SW3 osteocytes (day 21) after 4 freezing and thawing cycles. In addition, IDG-SW3 osteocytes, plated at a density of $4 \times 10^{4}$ cells $/ \mathrm{cm}^{2}$, were cultured on day 21 of differentiation for 24 hours with FCS (viable), without FCS (starved), or for 12 hours with $1 \mu \mathrm{M}$ dexamethasone (MilliporeSigma) (apoptotic). Afterwards, the medium was exchanged with FCS-containing medium in all conditions and collected after 24 hours for SAP-130 measurements, according to the manufacturer's instructions (Biorbyt).

Murine osteoclast differentiation. Total BM cells from WT and Mincle-KO mice were isolated by flushing the femur and tibia. The cells were plated overnight at $37^{\circ} \mathrm{C}$ and $5.5 \% \mathrm{CO}_{2}$ in a $100 \times 20 \mathrm{~mm}$ dish in osteoclast medium, composed of $\alpha \mathrm{MEM}$ and GlutaMAX (Gibco) with $10 \%$ FCS and 1\% penicillin/streptomycin (Gibco), supplemented with $5 \mathrm{ng} / \mathrm{mL}$ M-CSF (PeproTech). The next day, nonadherent BMMs were collected, washed, and further cultured in osteoclast medium with 20 $\mathrm{ng} / \mathrm{mL} \mathrm{M}-\mathrm{CSF}$ and $10 \mathrm{ng} / \mathrm{mL}$ RANKL (PeproTech) in 96-well plates (TRAP; $200 \mu \mathrm{L} /$ well) or 48 -well plates (RNA; $500 \mu \mathrm{L} /$ well) at the concentration of $1 \times 10^{6}$ cells $/ \mathrm{mL}$ at $37^{\circ} \mathrm{C}$ and $5.5 \% \mathrm{CO}_{2}$. The medium was changed every 2 days. In some experiments, preosteoclasts were further stimulated on day 1 of culture with the following stimulants: TDB (MilliporeSigma), neutralizing anti-mMincle IgG (InvivoGen, mabg-mmcl), rat IgG2b isotype control (R\&D Systems, MAB0061), synthetic $\beta$-GlcCer [d18:1, C24:1(15Z), Avanti Polar Lipids], apoptotic and necrotic splenocytes, viable and necrotic osteocytes, and supernatants from viable and necrotic osteocytes. Fully differentiated osteoclasts (day 4-5) were washed with PBS, fixed with fixation buffer (MilliporeSigma), and stained with TRAP solution.

Immunofluorescence in osteoclasts. For immunofluorescence, $1 \times 10^{6}$ BMMs/well were cultured in $1 \mathrm{~mL} /$ well osteoclast medium with supplements on 12-mm circle coverslips (Thermo Fisher Scientific) in 24-well plates at $37^{\circ} \mathrm{C}$ and $5.5 \% \mathrm{CO}_{2}$ until fully differentiated. The medium was changed every 2 days. After fixation, the cells were stained with
Acti-stain 670 phalloidin (Cytoskeleton, Inc.) according to the manufacturer's instructions to visualize F-actin ring formation. Cells were also stained with fluorescence-based TRAP staining, using ELF 97 phosphatase substrate (Invitrogen) as described previously (65) in combination with anti-Mincle antibody, which was used as indicated above.

Resorption assay. For the resorption assay, $1 \times 10^{6} \mathrm{BMMs} /$ well were cultured in 24-well plates coated with calcium phosphate (Corning) in $1 \mathrm{~mL} /$ well osteoclast medium with $20 \mathrm{ng} / \mathrm{mL} \mathrm{M-CSF}$ and $30 \mathrm{ng} / \mathrm{mL}$ RANKL at $37^{\circ} \mathrm{C}$ and $5.5 \% \mathrm{CO}_{2}$. The medium was changed every 2 days. After 5 days of differentiation, the osteoclasts were lysed with $\mathrm{dH}_{2} \mathrm{O}$ and the plates were incubated with $5 \%$ sodium hypochlorite (MilliporeSigma) for 5 minutes. Afterwards, the plates were washed and dried for 5 hours at room temperature.

All images were acquired with the Keyence fluorescence microscope and quantification of osteoclast number and percentage of the resorbed area was performed with ImageJ.

RNA-seq. Osteoclast precursors from WT or Mincle-KO mice were stimulated on day 1 of culture for 24 hours with supernatant from necrotic osteocytes (1:2) (CtNecOt, KONecOt) compared with control condition (supernatant from viable osteocytes 1:2) (Ct, KO). Total RNA was harvested on day 3 of culture. RNA-seq was carried out by Novogene. A total amount of $1 \mu \mathrm{g}$ RNA per sample was used as input material for the RNA sample preparations. Sequencing libraries were generated using the NEBNext Ultra RNALibrary Prep Kit for Illumina (New England Biolabs). Clustering of the index-coded samples was completed on a cBot Cluster Generation System using PE Cluster Kit cBot-HS (Illumina), according to the manufacturer's instructions. The library preparations were sequenced on an Illumina HiSeq platform and paired-end reads were generated. The filtered reads were aligned to the Mus musculus genome (Mus_musculus.GRCm38) with HISAT2 (version 2.0.5). HTSeq (version 0.6.1) was used to count the read numbers mapped of each gene, including known and novel genes. Differential expression analysis between 2 conditions or groups (3 biological replicates per condition) was performed using DESeq2 R package (version 1.20.0). The resulting $P$ values were adjusted using the Benjamini-Hochberg approach for controlling the false discovery rate (FDR). Genes with an adjusted $P$ value $\left(P_{\text {adj }}\right)$ less than 0.05 were assigned as differentially expressed. KEGG pathway enrichment analysis was performed with KOBAS software (version 3.0); pathway $P_{\text {adj }}$ values less than 0.05 were considered significantly enriched. All original RNA-seq data were deposited in the NCBI's Gene Expression Omnibus database (GEO GSE151049).

Extracellular flux assay using the Seahorse platform. For metabolic analysis, $2 \times 10^{5} \mathrm{BMMs} /$ well were plated on Seahorse XF96 Cell Culture Microplates (Agilent) in $200 \mu \mathrm{L} /$ well osteoclast medium with supplements at $37^{\circ} \mathrm{C}$ and $5.5 \% \mathrm{CO}_{2}$. On day 1 of culture, cells were stimulated with a 1:2 ratio of necrotic osteocyte supernatant for 24 hours until medium change. On day 3 of culture, mitochondrial respiration (Seahorse XF Cell Mito Stress Test) using final concentrations of $2 \mu \mathrm{M}$ oligomycin, $2 \mu \mathrm{M}$ carbonyl cyanide 4 -(trifluoromethoxy) phenylhydrazone (FCCP), $1 \mu \mathrm{M}$ antimycin A, and $1 \mu \mathrm{M}$ rotenone (all from MilliporeSigma) was measured in preosteoclasts, according to the manufacturer's instructions. Oxygen consumption rate (OCR) was measured in a Seahorse XFe96 Analyzer (Agilent) and the values were normalized to the protein concentration within the single wells, detected with the DC protein assay (Bio-Rad). Data were analyzed with the Seahorse XF Report Generator for Mito Stress Test. 
TEM. BMMs $\left(1 \times 10^{6} /\right.$ well $)$ were cultured in $1 \mathrm{~mL} /$ well osteoclast medium on 13-mm Thermanox plastic coverslips (Thermo Fisher Scientific) in 24 -well plates at $37^{\circ} \mathrm{C}$ and $5.5 \% \mathrm{CO}_{2}$. The medium was changed every 2 days. On day 1 of culture, cells were stimulated with necrotic osteocytes (1:2). When the osteoclasts were fully differentiated, they were washed with PBS and fixed with $2.5 \%$ glutaraldehyde in $0.1 \mathrm{M}$ phosphate buffer for at least 48 hours. Thereupon, the cells were postfixed in $2 \%$ buffered osmium tetroxide for 2 hours and then dehydrated in graded alcohol concentrations and embedded in epoxy resin, according to standard protocols. For orientation, 1- $\mu \mathrm{m}$ semithin sections were stained with toluidine blue. Ultrathin sections were stained with uranyl acetate and lead citrate and examined with a transmission electron microscope (EM 906E, Carl Zeiss).

Movement tracking. BMMs $\left(2.5 \times 10^{5} /\right.$ well $)$ from WT or Mincle-KO mice were cultured in 48 -well plates in $500 \mu \mathrm{L} /$ well osteoclast medium with $20 \mathrm{ng} / \mathrm{mL} \mathrm{M-CSF}$ and $10 \mathrm{ng} / \mathrm{mL}$ RANKL at $37^{\circ} \mathrm{C}$ and $5.5 \%$ $\mathrm{CO}_{2}$. The medium was changed once on day 2 . On day 2 , the cells were additionally stimulated with necrotic osteocytes (1:2) and immediately placed in the Keyence incubation chamber at $37^{\circ} \mathrm{C}$ and $5.5 \% \mathrm{CO}_{2}$. To analyze the motion parameters, total length $(\mu \mathrm{m})$, speed $(\mu \mathrm{m} / \mathrm{min})$, distance $(\mu \mathrm{m})$, and motion angle (degrees), the live cell movement was monitored over a time period of 500 minutes (recording every 5 $\mathrm{min} / 100$ images in total) with the Keyence microscope in preselected spots. To remove out-of-focus signals, $Z$-stacks were acquired for each spot. Afterwards, the images were stitched together and processed with ImageJ. Movement analyses and 3D track plots were carried out with the free software CellTracker (66).

Human osteoclast differentiation. Human peripheral blood mononuclear cells (PBMCs) were isolated from EDTA-blood of normal, healthy donors, using a Ficoll gradient (Lymphoflot, Bio-Rad). PBMCs $\left(3 \times 10^{5} /\right.$ well $)$ were plated in 96 -well plates (TRAP) or $7.5 \times 10^{5} \mathrm{PBMCs} /$ well in 48 -well plates (RNA) in $100 \mu \mathrm{L} /$ well or $250 \mu \mathrm{L} /$ well osteoclast medium (1\% FCS and $1 \%$ pen/strep), respectively, for $1-2$ hours at $37^{\circ} \mathrm{C}$ and $5.5 \% \mathrm{CO}_{2}$ to purify monocytes by plastic adhesion. Afterwards, the cells were washed and differentiated into osteoclasts in $200 \mu \mathrm{L} /$ well or $500 \mu \mathrm{L} /$ well osteoclast medium (10\% FCS and 1\% pen/strep) with $30 \mathrm{ng} / \mathrm{mL}$ M-CSF, $2 \mathrm{ng} / \mathrm{mL}$ RANKL, and $1 \mathrm{ng} / \mathrm{mL}$ TGF- $\beta$ (all PeproTech) at $37^{\circ} \mathrm{C}$ and $5.5 \% \mathrm{CO}_{2}$. Two-thirds of the medium was changed on day 3 and again on day 5 of differentiation. After 5 days, preosteoclasts were incubated as indicated with TDB or necrotic cells, generated by 4 freezing and thawing cycles of the remaining PBMCs. Osteoclast differentiation was evaluated by TRAP staining, when osteoclasts were fully differentiated (day 7). Images were acquired with the Keyence microscope and quantification of osteoclast number was performed with ImageJ.

RNA extraction and quantitative real-time PCR. RNA from murine and human osteoclasts was isolated with peqGOLD TRIfast (Peqlab) according to standard protocols. Human jawbone samples were homogenized and RNA was purified with the RNeasy Mini Kit (QIAGEN) according to the manufacturer's instructions. RNA was reversely transcribed with the High-Capacity cDNA Reverse Transcription Kit (Applied Biosystems). SYBR Green-based quantitative real-time PCR was performed on a CFX96 Touch Real-Time PCR Detection System (Bio-Rad). Samples were analyzed in duplicate and normalized to the level of $A c t b$ ( $\beta$-actin) mRNA for murine and B2M ( $\beta$-2-microglobulin) mRNA for human samples. Human and murine qPCR primer sequences are listed in Supplemental Tables 1 and 2, respectively.
Patients. Jawbone specimens from patients with MRONJ were collected intraoperatively during sequestrotomy as part of the surgical therapy for MRONJ at the Department of Oral and Maxillofacial Surgery at the Universitätsklinikum Erlangen. Bone specimens were fixed in $4 \%$ PFA immediately after extraction. For realtime PCR, bone specimens were stored in RNAlater (QIAGEN). Routine histopathological diagnostics were performed with each of the specimens at the Department of Pathology of the Universitätsklinikum Erlangen to confirm the diagnosis of MRONJ. The control specimens were obtained during dental surgery and modeling osteotomy of bone reconstructive surgical procedures. The control specimens were clinically free of inflammation and free of any antiresorptive or radiotherapy exposure.

Osteonecrotic lesions from patients with ONFH were recruited from the Department of Orthopaedic Surgery of the University Medical Center Hamburg-Eppendorf. Bone specimens were fixed in $4 \%$ PFA immediately after extraction. All of these patients fulfilled the clinical and radiological criteria for ONFH.

For additional information, see Supplemental Methods.

Statistics. All statistical analyses were performed using GraphPad Prism v8. Data are presented as mean \pm SD. Statistical significance was calculated by 2-tailed Student's $t$ test for 2-group comparisons and 1-way or 2-way ANOVA for multiple comparisons. Correlations were tested with the linear regression $F$ test. $P$ values are given exactly.

Study approval. All analyses of human material were performed in accordance with the institutional guidelines and with the approval of the ethics committee of the Universitätsklinikum Erlangen and University Medical Center Hamburg-Eppendorf. All experiments with animals were authorized by the ethics committee of the Government of Lower Franconia. Animals were kept following the guidelines of the German Animal Welfare Act.

\section{Author contributions}

DA, GS, and AB designed the study. DA, ML, DW, KK, MF, AG, USS, and LEM acquired the data. DA, US, BG, FW, GK, RL, GS, and $\mathrm{AB}$ interpreted the data. US, BK, AML, AN, FW, and RL provided material support. RL provided the Mincle-KO mice. DA, GS, and $\mathrm{AB}$ prepared the manuscript. GS and $\mathrm{AB}$ acquired funding.

\section{Acknowledgments}

We thank Erwin F. Wagner (MedUni Wien, Vienna) for providing the IDG-SW3 cell line. The authors thank W. Baum for teaching and providing the instruments for the 3-point bending fracture model and C. Zech, B. Happich, H. Symowski, and N. Berndt for excellent technical assistance. In addition, we wish to thank the Optical Imaging Centre Erlangen (OICE) within the Z project of the CRC 1181 for support with imaging. This study was supported by the Collaborative Research Centre (CRC) 1181 project-A01; the Deutsche Forschungsgemeinschaft (DFG, German Research Foundation) BO-3811/5-1, BO-3811/6-1, and FOR2886 TP02; the SPP $\mu$ Bone; the Interdisciplinary Center for Clinical Research (IZKF) grant A77 and J76; and the European Research Council (ERC) Synergy Grant 4D Nanoscope.

Address correspondence to: Aline Bozec, Glückstraße 6, 91054 Erlangen, Germany. Phone: 49.9131.85.29002; Email: aline. bozec@uk-erlangen.de. 
1. Boyle WJ, Simonet WS, Lacey DL. Osteoclast differentiation and activation. Nature. 2003;423(6937):337-342.

2. Teitelbaum SL, Ross FP. Genetic regulation of osteoclast development and function. Nat Rev Genet. 2003;4(8):638-649.

3. Xiong J, Onal M, Jilka RL, Weinstein RS, Manolagas SC, O'Brien CA. Matrix-embedded cells control osteoclast formation. Nat Med. 2011;17(10):1235-1241.

4. Nakashima T, et al. Evidence for osteocyte regulation of bone homeostasis through RANKL expression. Nat Med. 2011;17(10):1231-1234.

5. Bonewald LF. The amazing osteocyte. JBone Miner Res. 2011;26(2):229-238.

6. Zimmerman SM, et al. Loss of RANKL in osteocytes dramatically increases cancellous bone mass in the osteogenesis imperfecta mouse (oim). Bone Rep. 2018;9:61-73.

7. Zhang B, et al. Mechanically induced autophagy is associated with ATP metabolism and cellular viability in osteocytes in vitro. Redox Biol. 2018;14:492-498.

8. Verborgt O, Gibson GJ, Schaffler MB. Loss of osteocyte integrity in association with microdamage and bone remodeling after fatigue in vivo. J Bone Miner Res. 2000;15(1):60-67.

9. Jilka RL, Noble B, Weinstein RS. Osteocyte apoptosis. Bone. 2013;54(2):264-271.

10. Komori T. Cell death in chondrocytes, osteoblasts, and osteocytes. Int J Mol Sci. 2016;17(12):E2045.

11. Böhm C, et al. RSK2 protects mice against TNF-induced bone loss. J Cell Sci. 2012;125(pt 9):2160-2171.

12. Foell D, Wittkowski H, Roth J. Mechanisms of disease: a 'DAMP' view of inflammatory arthritis. Nat Clin Pract Rheumatol. 2007;3(7):382-390.

13. Noble BS, Reeve J. Osteocyte function, osteocyte death and bone fracture resistance. Mol Cell Endocrinol. 2000;159(1-2):7-13.

14. Zhang Q, et al. Circulating mitochondrial DAMPs cause inflammatory responses to injury. Nature. 2010;464(7285):104-107.

15. Weinstein RS, Nicholas RW, Manolagas SC. Apoptosis of osteocytes in glucocorticoid-induced osteonecrosis of the hip. JClin Endocrinol Metab. 2000;85(8):2907-2912.

16. Takeuchi O, Akira S. Pattern recognition receptors and inflammation. Cell. 2010;140(6):805-820.

17. Roh JS, Sohn DH. Damage-associated molecular patterns in inflammatory diseases. Immune Netw. 2018;18(4):e27.

18. Patin EC, Orr SJ, Schaible UE. Macrophage inducible C-type lectin as a multifunctional player in immunity. Front Immunol. 2017;8:861.

19. Ishikawa E, et al. Direct recognition of the mycobacterial glycolipid, trehalose dimycolate, by C-type lectin Mincle. J Exp Med. 2009;206(13):2879-2888.

20. Schoenen $\mathrm{H}$, et al. Cutting edge: Mincle is essential for recognition and adjuvanticity of the mycobacterial cord factor and its synthetic analog trehalose-dibehenate. Jimmunol. 2010;184(6):2756-2760.

21. Patin EC, Willcocks S, Orr S, Ward TH, Lang R, Schaible UE. Mincle-mediated anti-inflammatory IL-10 response counter-regulates IL-12 in vitro. Innate Immun. 2016;22(3):181-185.

22. Yamasaki S, Ishikawa E, Sakuma M, Hara H, Ogata K, Saito T. Mincle is an ITAM-coupled activating receptor that senses damaged cells. Nat Immunol. 2008;9(10):1179-1188.

23. Nagata $\mathrm{M}$, et al. Intracellular metabolite $\beta$-glucosylceramide is an endogenous Mincle ligand possessing immunostimulatory activity. Proc Natl Acad Sci U S A. 2017;114(16):E3285-E3294.

24. Drouin M, Saenz J, Chiffoleau E. C-type lectinlike receptors: head or tail in cell death immunity. Frontiers in immunology. 2020;11:251.

25. Tatsumi S, et al. Targeted ablation of osteocytes induces osteoporosis with defective mechanotransduction. Cell Metab. 2007;5(6):464-475.

26. Feng JQ, et al. Loss of DMP1 causes rickets and osteomalacia and identifies a role for osteocytes in mineral metabolism. Nat Genet. 2006;38(11):1310-1315.

27. Ling Y, Rios HF, Myers ER, Lu Y, Feng JQ, Boskey AL. DMP1 depletion decreases bone mineralization in vivo: an FTIR imaging analysis. JBone Miner Res. 2005;20(12):2169-2177.

28. Brown GD. Sensing necrosis with Mincle. Nat Immunol. 2008;9(10):1099-1100.

29. Harre U, et al. Moonlighting osteoclasts as undertakers of apoptotic cells. Autoimmunity. 2012;45(8):612-619.

30. Grüneboom A, et al. A network of trans-cortica capillaries as mainstay for blood circulation in long bones. Nat Metab. 2019;1(2):236-250.

31. Kalu DN. The ovariectomized rat model of postmenopausal bone loss. Bone Miner. 1991;15(3):175-191.

32. Appel H, et al. Altered skeletal expression of sclerostin and its link to radiographic progression in ankylosing spondylitis. Arthritis Rheum. 2009;60(11):3257-3262.

33. Korganow AS, et al. From systemic T cell self-reactivity to organ-specific autoimmune disease via immunoglobulins. Immunity. 1999;10(4):451-461.

34. Einhorn TA, Gerstenfeld LC. Fracture healing: mechanisms and interventions. Nat Rev Rheuma tol. 2015;11(1):45-54.

35. Cheung WY, et al. Pannexin-1 and P2X7-receptor are required for apoptotic osteocytes in fatigued bone to trigger RANKL production in neighboring bystander osteocytes. J Bone Miner Res. 2016;31(4):890-899.

36. Zong WX, Thompson CB. Necrotic death as a cell fate. Genes Dev. 2006;20(1):1-15.

37. Takayanagi $\mathrm{H}$. Interaction between the immune system and bone metabolism: an emerging field of osteoimmunology. Proc Jpn Acad, Ser B, Phys Biol Sci. 2007;83(5):136-143.

38. Ishii KA, et al. Coordination of PGC-1beta and iron uptake in mitochondrial biogenesis and osteoclast activation. Nat Med. 2009;15(3):259-266.

39. Kim H, et al. Tmem64 modulates calcium signaling during RANKL-mediated osteoclast differentiation. Cell Metab. 2013;17(2):249-260.

40. Jin Z, Wei W, Yang M, Du Y, Wan Y. Mitochondrial complex I activity suppresses inflammation and enhances bone resorption by shifting macrophage-osteoclast polarization. Cell Metab. 2014;20(3):483-498.

41. Emerton KB, et al. Osteocyte apoptosis and con- trol of bone resorption following ovariectomy in mice. Bone. 2010;46(3):577-583.

42. Tomkinson A, Reeve J, Shaw RW, Noble BS. The death of osteocytes via apoptosis accompanies estrogen withdrawal in human bone. J Clin Endocrinol Metab. 1997;82(9):3128-3135.

43. Hayashi M, Nakashima T, Yoshimura N, Okamoto K, Tanaka S, Takayanagi H. Autoregulation of osteocyte Sema3A orchestrates estrogen action and counteracts bone aging. Cell Metab. 2019;29(3):627-637.e5.

44. Manolagas SC, O'Brien CA, Almeida M. The role of estrogen and androgen receptors in bone health and disease. Nat Rev Endocrinol. 2013;9(12):699-712.

45. Wu Y, et al. Bone microenvironment specific roles of ITAM adapter signaling during bone remodeling induced by acute estrogen-deficiency. PLoS One. 2007;2(7):e586.

46. Badros A, et al. Natural history of osteonecrosis of the jaw in patients with multiple myeloma. JClin Oncol. 2008;26(36):5904-5909.

47. Moya-Angeler J, Gianakos AL, Villa JC, Ni A, Lane JM. Current concepts on osteonecrosis of the femoral head. World JOrthop. 2015;6(8):590-601.

48. Wang C, et al. Bone microstructure and regional distribution of osteoblast and osteoclast activity in the osteonecrotic femoral head. PLoS One. 2014;9(5):e96361.

49. Kamiya N, Yamaguchi R, Aruwajoye O, Adapala NS, Kim HK. Development of a mouse model of ischemic osteonecrosis. Clin Orthop Relat Res. 2015;473(4):1486-1498.

50. Zhou L, et al. COMP-angiopoietin1 potentiates the effects of bone morphogenic protein-2 on ischemic necrosis of the femoral head in rats. PLoS One. 2014;9(10):e110593.

51 . Wang C, et al. Analysis of early stage osteonecrosis of the human femoral head and the mechanism of femoral head collapse. Int J Biol Sci. 2018;14(2):156-164.

52. Favia G, Pilolli GP, Maiorano E. Histologic and histomorphometric features of bisphosphonate-related osteonecrosis of the jaws: an analysis of 31 cases with confocal laser scanning microscopy. Bone. 2009;45(3):406-413.

53. Bedogni A, Bettini G, Totola A, Saia G, Nocini PF. Oral bisphosphonate-associated osteonecrosis of the jaw after implant surgery: a case report and literature review. JOral Maxillofac Surg. 2010;68(7):1662-1666.

54. Bi Y, et al. Bisphosphonates cause osteonecrosis of the jaw-like disease in mice. Am J Pathol. 2010;177(1):280-290.

55. Córdova LA, et al. Severe compromise of preosteoblasts in a surgical mouse model of bisphosphonate-associated osteonecrosis of the jaw. JCraniomaxillofac Surg. 2016;44(9):1387-1394.

56. Gross C, et al. Osteoclast profile of medication-related osteonecrosis of the jaw secondary to bisphosphonate therapy: a comparison with osteoradionecrosis and osteomyelitis. J Transl Med. 2017;15(1):128.

57. Wells CA, et al. The macrophage-inducible C-type lectin, mincle, is an essential component of the innate immune response to Candida albicans. J Immunol. 2008;180(11):7404-7413.

58. Lu Y, Xie Y, Zhang S, Dusevich V, Bonewald 
LF, Feng JQ. DMP1-targeted Cre expression in odontoblasts and osteocytes. JDent Res. 2007;86(4):320-325.

59. Buch T, et al. A Cre-inducible diphtheria toxin receptor mediates cell lineage ablation after toxin administration. Nat Methods. 2005;2(6):419-426.

60. Chen Z, et al. Th2 and eosinophil responses suppress inflammatory arthritis. Nat Commun. 2016;7:11596.

61. Hiltunen A, Vuorio E, Aro HT. A standardized experimental fracture in the mouse tibia. JOrthop Res. 1993;11(2):305-312.

62. Klingberg A, et al. Fully automated evaluation of total glomerular number and capillary tuft size in nephritic kidneys using lightsheet microscopy. J Am Soc Nephrol. 2017;28(2):452-459.

63. Schmid B, et al. 3Dscript: animating 3D/4D microscopy data using a natural-language-based syntax. Nat Methods. 2019;16(4):278-280.

64. Woo SM, Rosser J, Dusevich V, Kalajzic I, Bonewald LF. Cell line IDG-SW3 replicates osteo- blast-to-late-osteocyte differentiation in vitro and accelerates bone formation in vivo. J Bone Miner Res. 2011;26(11):2634-2646.

65. Filgueira L. Fluorescence-based staining for tartrate-resistant acidic phosphatase (TRAP) in osteoclasts combined with other fluorescent dyes and protocols. J Histochem Cytochem. 2004;52(3):411-414.

66. Piccinini F, Kiss A, Horvath P. CellTracker (not only) for dummies. Bioinformatics. 2016;32(6):955-957. 